\title{
Excursion Measure Away from an Exit Boundary of One-Dimensional Diffusion Processes
}

\author{
By
}

Kouji YANO*

\begin{abstract}
A generalization of the excursion measure away from an exit boundary is defined for a one-dimensional diffusion process. It is constructed through the disintegration formula with respect to the lifetime. The counterpart of the Williams description, the disintegration formula with respect to the maximum, is also established. This generalized excursion measure is applied to explain and generalize the convergence theorem of Kasahara and Watanabe [8] in terms of the Poisson point fields, where the inverse local time processes of regular diffusion processes converge in the sense of probability law to some Lévy process, which is closely related to a diffusion process with an exit boundary.
\end{abstract}

\section{$\S 1 . \quad$ Introduction}

Watanabe [18] has discovered the necessary and sufficient condition that the ratio of the occupation time on the positive side of a one-dimensional generalized diffusion process converges in law to some non-trivial random variable. In the positively recurrent cases, in particular, the limit random variable is a constant.

Recently Kasahara and Watanabe [8] have studied the scaling limit of the fluctuation in the positively recurrent cases. In their context, they obtained the following convergence theorem: The renormalized inverse local time processes

Communicated by Y. Takahashi. Received December 9, 2005.

2000 Mathematics Subject Classification(s): 60J60 (60F05, 60G55, 60G52, 34L05, 34B24).

Key words: diffusion process, excursion theory, Poisson point process, limit theorem, stable process, Krein's spectral theory

*RIMS, Kyoto University, Kyoto 606-8502, Japan.

e-mail: yano@kurims.kyoto-u.ac.jp 
at the origin converge in law to some Lévy process which is not necessarily a subordinator. Indeed the corresponding strings for which the origin is a regular boundary converge to a string for which the origin is an exit boundary. The notion of this convergence, which was introduced in Kasahara-Watanabe [8] and Kotani [12], is a breakthrough in this problem. We state its definition in Definition 3.1.

We consider non-singular conservative $\frac{d}{d m} \frac{d}{d x}$-diffusion processes and generalize the convergence theorem of Kasahara-Watanabe [8] in terms of the Poisson point fields. For this generalization we need to establish the generalized notion of the excursion measure $\boldsymbol{n}$ away from an exit boundary.

We have the following two well-known formulae of descriptions of usual excursion measures (see, e.g., [4] and [15]). One is the disintegration formula with respect to the lifetime $\zeta$ :

$$
\boldsymbol{n}(\Gamma)=\int_{0}^{\infty} \boldsymbol{P}_{t}^{0,0}(\Gamma) \boldsymbol{n}(\zeta \in d t) .
$$

The other is the disintegration formula with respect to the maximum $M$ :

$$
\boldsymbol{n}(\Gamma)=\int_{0}^{\infty} \boldsymbol{R}^{a}(\Gamma) \boldsymbol{n}(M \in d a)
$$

This is due to Williams [20] and is often called the Williams description. Here $\boldsymbol{P}_{t}^{0,0}$ and $\boldsymbol{R}^{a}$ are defined through the harmonic transform of the original process. We establish these two formulae (1.1) and (1.2) for our generalized excursion measures in Theorem 2.3 and Theorem 2.4 , respectively.

We consider a process defined by

$$
U[f](m ; t)=\int_{\{\zeta<1\}} f(\zeta(e)) \widetilde{\boldsymbol{N}}(m ;(0, t], d e)+\int_{\{\zeta \geq 1\}} f(\zeta(e)) \mathbf{N}(m ;(0, t], d e) .
$$

Here $\boldsymbol{N}(m ; d t, d e)$ and $\widetilde{\boldsymbol{N}}(m ; d t, d e)$ denote the Poisson point field with intensity measure $d t \boldsymbol{n}(d e)$ and its compensated random field, respectively. We establish the continuity theorem with respect to the string $m$, which is stated as Theorem 2.7:

$$
U[f]\left(m_{n} ; t\right) \stackrel{\text { law }}{\longrightarrow} U[f](m ; t)
$$

as $m_{n}$ converges to $m$ in the sense of Definition 3.1. If $f(x) \equiv x$, then the expression (1.3) gives the compensated inverse local time processes. Hence our continuity theorem (1.4) provides a generalization of the convergence theorem of Kasahara-Watanabe [8] in terms of the Poisson point fields. 
The essence of the proof of the existence theorem of the generalized excursion measures lies in Proposition 2.1, which asserts that an entrance law exists. Its density with respect to $d m(x)$ is given by the partial derivative $\Pi(t, x)$ of $q(t, x, y)$ at $y=0+$. Here $q(t, x, y)$ denotes the transition probability for which the origin is an absorbing boundary. Proposition 2.1 allows us to interchange the differentiation and the integration in the eigendifferential expansion

$$
q(t, x, y)=\int_{(0, \infty)} e^{-t \xi} \psi_{-\xi}(x) \psi_{-\xi}(y) \theta(d \xi) .
$$

We must be careful in interchanging the differentiation and the integration for such an eigendifferential expansion. For instance, we consider the eigendifferential expansion of the resolvent kernel:

$$
G(\lambda, x, y)=\int_{(0, \infty)} \frac{\psi_{-\xi}(x) \psi_{-\xi}(y)}{\lambda+\xi} \theta(d \xi) .
$$

Then it can never hold that

$$
\left.\frac{\partial^{2} G}{\partial x \partial y}(\lambda, x, y)\right|_{y=x}=\int_{(0, \infty)} \frac{\left|\psi_{-\xi}^{\prime}(x)\right|^{2}}{\lambda+\xi} \theta(d \xi) .
$$

In fact, the LHS equals to the product of the derivatives of the positive increasing eigenfunction and the decreasing one with eigenvalue $\lambda$. This means that the LHS of (1.7) is negative, while the RHS of (1.7) is obviously positive. Hence the identity (1.7) fails.

The foundation of the excursion theory is established by Itô [5]. (We can find it in standard textbooks, e.g., [4] and [15]. See also [2] in a general framework.) Consider the inverse local time process $(\eta(t))$ for a diffusion process at a regular point, say, the origin. Then it is an increasing Lévy process, namely, a subordinator. To each jump of the process $(\eta(t))$ we assign a piece of the path starting from the origin and coming back there, called an excursion away from the origin. Then we obtain a point process $(p(t))$. Denote the counting measure of $(p(t))$ by $\boldsymbol{N}(d t, d e)$. Then the process $(\eta(t))$ admits an integral expression

$$
\eta(t)=\int \zeta(e) \boldsymbol{N}((0, t], d e) .
$$

The strong Markov property together with the time homogeneity of the diffusion process assures that $(p(t))$ forms a stationary Poisson point process and that $\mathbf{N}(d t, d e)$ a Poisson point field. The law of $\boldsymbol{N}(d t, d e)$ is characterized by its intensity measure $d t \boldsymbol{n}(d e)$, where $\boldsymbol{n}$ is a $\sigma$-finite measure defined on the 
space of excursions away from the origin. The measure $\boldsymbol{n}$ is called the excursion measure away from the origin of the diffusion process.

Based on Krein's spectral theory (see, e.g., [3], [7] and [13]), Knight [10] and Kotani-Watanabe [13] have characterized the class of the Lévy measures of $(\eta(t))$ for one-dimensional generalized (or gap) diffusion processes. For a string $m$, the corresponding Lévy measure has a density $\rho(u)=\int_{(0, \infty)} e^{-u \xi} \xi \sigma^{*}(d \xi)$ where $\sigma^{*}$ is the spectral measure of the dual string $m^{*}$. This fact is extremely useful for investigating the law of the occupation time. Watanabe's result [18], mentioned in the beginning of this section, was based on this fact (see also [1], [9], [21] and [19]).

We may say that Kasahara and Watanabe ([8]) have generalized these results. They showed that any string $m$ for which the origin is of limit circle type corresponds to a Lévy process without Gaussian part nor negative jumps and characterized its Lévy measure by the spectral measure $\sigma^{*}$ of the dual string $m^{*}$. Their results are closely related to a recent work of Kotani [12], which gives a generalization of Krein's spectral theory. Some of their results will be stated in $\S 3.5$.

The key to our continuity theorem (Theorem 2.7) is to establish the following relation between two spectral measures $\theta$ and $\sigma^{*}$, stated in Theorem 2.2:

$$
\theta(d \xi)=\xi \sigma^{*}(d \xi)
$$

This result unifies the framework of our generalized excursion measure in terms of $\theta$ with that of Knight [10], Kasahara-Watanabe [8] and Kotani [12] in terms of $\sigma^{*}$.

The present paper is organized as follows. In $\S 2$, we will state our results after a brief review of the known results. In $\S 3$ and $\S 4$, we prepare some notations and some preliminary results for the eigendifferential expansion at an exit boundary of the fundamental solution for operators of the form $\frac{d}{d m} \frac{d}{d x}$ and for the corresponding diffusion processes. In $\S 5$, we will introduce the $\sigma$ fields which represent the information of the path on the intervals between two random times. We need a careful treatment of them to establish the generalized Williams description. In $\S 6$, we prove the existence theorem of the excursion measure away from an exit boundary for absorbing $\mathcal{L}_{m}$-diffusion process, which we denote by $\boldsymbol{n}$. We will construct it through the disintegration formula with respect to the lifetime. In $\S 7$, we will prove the generalized Williams description for our excursion measure. For this, we establish the strong Markov property and the first-entrance-last-exit decomposition for $\boldsymbol{n}$. For the proofs we fully 
utilize the results in $\S 5$. $\S 8$ is devoted to the proof of the continuity theorem, Theorem 2.7. From this we can derive Corollary 2.6, i.e. the convergence of the processes defined by integrals with respect to Poisson point fields, which generalize the convergence theorem of Kasahara-Watanabe [8].

Notation. Throughout this paper, the integration (or expectation) with respect to a positive measure $\boldsymbol{m}(\cdot)$ on a path space is denoted by $\boldsymbol{m}[\cdot]$.

\section{$\S 2 . \quad$ Results}

\section{$\S 2.1$. The background}

To explain our motivation, we shall make a brief review of the known results.

Let $m:[0, \infty) \rightarrow[0, \infty)$ be a string with $m(0)=0$. Then there corresponds a $\frac{d}{d m} \frac{d}{d x}$-diffusion process for which the origin is a reflecting boundary. Denote its inverse local time process at the origin by $(\eta(t))$. Then the process $(\eta(t))$ is a subordinator whose law has the Laplace transform given by

$$
\boldsymbol{E}[\exp (-s \eta(t))]=\exp (-t \Psi(s)), \quad s>0, t>0
$$

where the exponent $\Psi(s)$ is given as

$$
\Psi(s)=\int_{0}^{\infty}\left(1-e^{-s u}\right) \rho(u) d u, \quad s>0
$$

with

$$
\rho(u)=\int_{(0, \infty)} e^{-u \xi} \xi \sigma^{*}(d \xi), \quad u>0 .
$$

Suppose that $m(x)$ is regularly varying at $x=\infty$, i.e., there exist a constant $\beta \in(0, \infty)$ and a slowly varying function $L(x)$ such that

$$
m(x) \sim x^{\beta} L(x), \quad x \rightarrow \infty .
$$

Then we have the following convergence in law:

$$
\eta_{\lambda}(t):=\frac{1}{\lambda^{\frac{1}{\alpha}} L(\lambda)} \eta(\lambda t) \stackrel{\text { law }}{\longrightarrow} \eta^{(\alpha)}(t), \quad \lambda \rightarrow \infty
$$

where $\alpha=1 /(1+\beta) \in(0,1)$ and $\eta^{(\alpha)}(t)$ is an $\alpha$-stable subordinator. This can be easily verified since $\left(\eta_{\lambda}(t)\right)$ is identical in law to the inverse local time process corresponding to a string $m_{\lambda}$ given by

$$
m_{\lambda}(x):=\frac{m(\lambda x)}{\lambda^{\frac{1}{\alpha}-1} L(\lambda)} \rightarrow x^{\frac{1}{\alpha}-1}, \quad \lambda \rightarrow \infty .
$$


In the positively recurrent cases, i.e., if $m(\infty)<\infty$, it holds that

$$
\frac{1}{\lambda} \eta(\lambda t) \stackrel{\text { law }}{\longrightarrow} m(\infty) t, \quad \lambda \rightarrow \infty .
$$

Hence it is natural to ask the scaling limit of the fluctuation

$$
\frac{1}{\lambda} \eta(\lambda t)-m(\infty) t
$$

Kasahara and Watanabe [8] answered this question.

Theorem 2.1 (Kasahara-Watanabe [8, Theorem 3.3]). Suppose that there exists a constant $\beta \in(0,1 / 2)$ and a slowly varying function $L(x)$ such that

$$
m(\infty)-m(x) \sim x^{-\beta} L(x), \quad x \rightarrow \infty
$$

Then, as $\lambda \rightarrow \infty$, it holds that

$$
\frac{1}{\lambda^{1 / \alpha-1} L(\lambda)}\left(\frac{1}{\lambda} \eta(\lambda t)-m(\infty) t\right) \stackrel{\text { law }}{\longrightarrow} T^{(\alpha)}(t),
$$

where $T^{(\alpha)}(t)$ is an $\alpha$-stable process with index $\alpha=1 /(1-\beta) \in(1,2)$.

We will generalize the convergence (2.10) as Corollary 2.6, which is stated in terms of the Poisson point fields.

Remark 1. Let $m(x)$ be a string which satisfies the assumptions of Theorem 2.1. Set

$$
m_{\lambda}(x)=\frac{1}{\lambda^{1 / \alpha-1} L(\lambda)}\{m(\lambda x)-m(\infty)\}
$$

and

$$
m^{(\alpha)}(x)=-x^{1 / \alpha-1}
$$

Then $m_{\lambda}$ converges to $m^{(\alpha)}$ in $\mathcal{M}_{1}$ as $\lambda \rightarrow \infty$. Here the definition of convergence in $\mathcal{M}_{1}$ is stated in Definition 3.1 .

\section{$\S 2.2$. Strings, operators and the classifications of boundaries}

In this subsection, we prepare the notations concerning strings, operators and the classifications of boundaries to state our theorems. 
Let $m(x)$ and $s(x)$ be two $(-\infty, \infty)$-valued non-decreasing functions on the interval $(r, l)$ with $-\infty \leq r<l \leq \infty$. We confine ourselves to the non-singular case, i.e.,

$$
m(x) \text { and } s(x) \text { are strictly increasing and continuous. }
$$

The functions $m(x)$ and $s(x)$ are identified with non-negative Radon measures $d m$ and $d s$ on $(r, l)$. The condition (2.13) is equivalent to the condition

$$
d m \text { and } d s \text { are everywhere positive and have no point masses. }
$$

We consider the second order differential operator

$$
\mathcal{L}_{(m, s)}=\frac{d}{d m} \frac{d}{d s} .
$$

If the scale $s(x) \equiv x$, then we denote $\mathcal{L}_{(m, s)}$ simply by $\mathcal{L}_{m}$ and call $m$ a string. (The self-adjoint extensions of $\mathcal{L}_{m}$ will be denoted by $L_{m}$ below.)

We follow Feller's theory of the classification of boundary points. Let

$$
c_{1}=\int_{\left(r, r^{\prime}\right]} d s(x) \int_{\left(x, r^{\prime}\right]} d m(y), \quad c_{2}=\int_{\left(r, r^{\prime}\right]} d m(x) \int_{\left(x, r^{\prime}\right]} d s(y)
$$

for some $r^{\prime} \in(r, l)$. Following Itô-McKean's book [6], we use the following terminology:

(i) If $c_{1}<\infty$, then the boundary $x=r$ is called exit.

(ii) If $c_{2}<\infty$, then the boundary $x=r$ is called entrance.

In particular, if it is both exit and entrance, then the boundary $x=r$ is called regular. Note that this classification is independent of the choice of $r^{\prime}$. The classification of the left boundary $x=l$ for $(m(x), s(x))$ is introduced as that of $x=-l$ for $(m(-x), s(-x))$ on the interval $(-l,-r)$.

Consider a string $m(x)$ on $(0, l)$ with $0<l \leq \infty$ (with the natural scale $s(x) \equiv x)$. Then

(i) The boundary $x=0$ is exit if and only if

$$
\int_{(0, \delta]} x d m(x)<\infty \quad \text { for some } \delta>0 .
$$

The class of such strings will be denoted by $\mathcal{M}$.

(ii) The boundary $x=0$ is of limit circle (Grenzkreis) type in the sense of Weyl's classification of the operator $\mathcal{L}_{m}=\frac{d}{d m} \frac{d}{d x}$ if and only if

$$
\int_{0}^{\delta} m(x)^{2} d x<\infty \quad \text { for some } \delta>0 .
$$


The class of such strings will be denoted by $\mathcal{M}_{1}$.

(iii) The boundary $x=0$ is regular if and only if

$$
m(0+)>-\infty \text {. }
$$

The class of such strings will be denoted by $\mathcal{M}_{0}$.

It is obvious that

$$
\mathcal{M}_{0} \subset \mathcal{M}_{1} \subset \mathcal{M}
$$

\section{$\S 2.3$. The fundamental solutions and the spectral measures}

Let $m \in \mathcal{M}$. We assume that the boundary $x=0$ is absorbing and that $x=l$ is also absorbing if $x=l$ is exit. Under these conditions, the operator $\mathcal{L}_{m}$ extends to a unique self-adjoint operator $L_{m}$ with its domain $\mathcal{D}\left(L_{m}\right)$.

Then we have the fundamental solution $q(t, x, y)$ of $L_{m}$ with eigendifferential expansion

$$
q(t, x, y)=\int_{(0, \infty)} e^{-t \xi} \psi_{-\xi}(x) \psi_{-\xi}(y) \theta(d \xi), \quad t>0, x, y \in(0, l) .
$$

The existence of the density of an entrance law is assured by the following proposition.

Proposition 2.1. $\quad$ Suppose that the spectral measure $\theta$ satisfies

$$
\int_{(0, \infty)} e^{-t \xi} \theta(d \xi)<\infty \quad \text { for any } t>0 .
$$

Then the following statements hold:

(i) For $t>0$ and $x \in(0, l)$, the function $q(t, x, y)$ is differentiable at $y=0$ and the partial derivative $\Pi(t, x)=\frac{\partial q}{\partial y}(t, x, 0+)$ satisfies

$$
\Pi(t, x)=\lim _{y \rightarrow 0+} \frac{q(t, x, y)}{y}=\int_{(0, \infty)} e^{-t \xi} \psi_{-\xi}(x) \theta(d \xi) .
$$

In particular, the function $\Pi(t, x)$ is non-negative.

(ii) The family of measures $\Pi(t, x) d m(x)$ defines an entrance law:

$$
\int_{(0, l)} \Pi(t, x) q(s, x, y) d m(x)=\Pi(t+s, y), \quad t, s>0, y \in(0, l) .
$$

(iii) The function $\Pi(t, x)$ is differentiable at $x=0$ and the derivative $\rho(t)=$ $\frac{\partial \Pi}{\partial x}(t, 0+)$ satisfies

$$
\rho(t)=\lim _{x \rightarrow 0+} \frac{\Pi(t, x)}{x}=\int_{(0, \infty)} e^{-t \xi} \theta(d \xi), \quad t>0 .
$$


The proof of Proposition 2.1 will be given in $§ 3.2$.

The following theorem gives the relation between the spectral measures $\theta$ and $\sigma^{*}$ (cf. (3.46) and (3.47) below).

Theorem 2.2. $\quad$ Let $m \in \mathcal{M}$. Suppose that the spectral measure $\sigma^{*}$ satisfies

$$
\int_{(0, \infty)} e^{-t \xi} \xi \sigma^{*}(d \xi)<\infty \quad \text { for any } t>0 .
$$

Then the condition $(\mathbf{S})$ holds and the following relation holds:

$$
\theta(d \xi)=\xi \sigma^{*}(d \xi) \quad \text { on }(0, \infty) .
$$

The proof of Theorem 2.2 will be given in $§ 3.4$.

Example 1. The assumption $\left(\mathbf{S}^{*}\right)$ (and hence the assumption $(\mathbf{S})$ ) is satisfied in the following cases:

(i) $m=m^{(\alpha)}$ for some $\alpha \in(0, \infty)$, where

$$
m^{(\alpha)}(x)= \begin{cases}x^{1 / \alpha-1}, & \text { if } \alpha \in(0,1), \\ \log x, & \text { if } \alpha=1, \\ -x^{1 / \alpha-1}, & \text { if } \alpha \in(1, \infty) .\end{cases}
$$

Indeed, the corresponding spectral measure $\sigma^{*}$ is given as $\sigma^{*}(d \xi)=C \xi^{\alpha} d \xi$ for some constant $C$. Note that the corresponding $L_{m}$-diffusion process is the Bessel process with index $-\alpha$ (or of dimension $2-2 \alpha \in(-\infty, 2)$ ).

(ii) $m \in \mathcal{M}_{1}$. Indeed, if $m \in \mathcal{M}_{1}$, then $\int_{[0, \infty)} \frac{\sigma^{*}(d \xi)}{1+\xi^{2}}<\infty$ (see Theorem 3.1 (i)).

Remark 2. In the case $m \in \mathcal{M}_{0}$, the relation (2.24) has been obtained by Minami-Ogura-Tomisaki [14, Lemma 3].

Remark 3. Kotani [11] has shown that there exists a (singular) string $m^{*}$ such that the corresponding spectral measure $\sigma^{*}$ satisfies

$$
\int_{(0, \infty)} e^{-t \xi} \sigma^{*}(d \xi)=\infty \quad \text { for any } t>0
$$

\section{§2.4. The excursion measures away from an exit boundary}

Let $m \in \mathcal{M}$ and suppose that the condition (S) is satisfied.

We give the precise definition of our excursion measure. Let $(E, \mathcal{E})$ denote the space of continuous paths with finite lifetime. Its precise definition will be given in $\S 4.3$. 
Definition 2.1. The excursion measure away from the origin of the $L_{m}$-diffusion process is a $\sigma$-finite measure $\boldsymbol{n}$ on the space $E$ such that

$$
\begin{aligned}
\boldsymbol{n}(C)= & \int_{A_{1}} d m\left(x_{1}\right) \Pi\left(t_{1}, x_{1}\right) \int_{A_{2}} d m\left(x_{2}\right) q\left(t_{2}-t_{1}, x_{1}, x_{2}\right) \\
& \cdots \int_{A_{n}} d m\left(x_{n}\right) q\left(t_{n}-t_{n-1}, x_{n-1}, x_{n}\right)
\end{aligned}
$$

for any cylinder set $C \in \mathcal{E}$ of the form

$$
C=\left\{e \in E: e\left(t_{1}\right) \in A_{1}, \ldots, e\left(t_{n}\right) \in A_{n}\right\} .
$$

This definition uniquely determines a measure on $\mathcal{E}$, if it exists, since $\mathcal{E}$ is generated by the totality of cylinder sets of the form (4.14). But it is needed to prove the existence of such a measure.

Let $\boldsymbol{P}_{t}^{0,0}$ denote the law of the pinned diffusion process of the harmonic transform of $L_{m}$ (cf. $\S 3.3$ and $\S 4.1$ ).

The following theorem assures the existence of the desired excursion measure and, at the same time, gives the disintegration formula with respect to the lifetime.

Theorem 2.3. $\quad$ Suppose that $m \in \mathcal{M}$ with $l=\infty$ and that the condition (S) is satisfied. Then the excursion measure $\boldsymbol{n}$ away from the origin of the $L_{m}$-diffusion exists and it possesses the following description:

$$
\boldsymbol{n}(\Gamma)=\int_{0}^{\infty} \boldsymbol{P}_{t}^{0,0}(\Gamma) \rho(t) d t, \quad \Gamma \in \mathcal{E}
$$

where

$$
\rho(t)=\int_{(0, \infty)} e^{-t \xi} d \theta(\xi)
$$

In particular, the excursion measure $\boldsymbol{n}$ is concentrated on $E^{0}=\{e \in E: e(0)=$ $0\}$.

The proof of Theorem 2.3 will be given in $\S 6$.

From this theorem we obtain the distribution of the lifetime $\zeta$ under the measure $\boldsymbol{n}$.

Corollary 2.1. $\quad$ Suppose that the assumption of Theorem 2.3 is satisfied. Then

$$
\boldsymbol{n}(\zeta \in A)=\int_{A} \rho(t) d t \quad \text { for any } A \in \mathcal{B}((0, \infty))
$$


Remark 4. We may interpret the disintegration formula (2.28) as the conditional distribution:

$$
\boldsymbol{n}(\Gamma \mid \zeta=t)=\boldsymbol{P}_{t}^{0,0}(\Gamma) \quad \text { for any } t>0 \text { and } \Gamma \in \mathcal{E} .
$$

By the symmetry of the transition kernel $p(t, x, y)$, the law $\boldsymbol{P}_{t}^{0,0}$ of the pinned $L_{m}^{h}$-diffusion process enjoys the time reversal property stated as

$$
\boldsymbol{P}_{t}^{0,0}\left(\Gamma^{\vee}\right)=\boldsymbol{P}_{t}^{0,0}(\Gamma), \quad \Gamma \in \mathcal{E}^{0} .
$$

Here the $\sigma$-field $\mathcal{E}^{0}$ and the time-several operator $(\cdot)^{\vee}$ will be introduced in (5.17) and (5.20), respectively. Applying this to the formula (2.28), we obtain the following.

Corollary 2.2 (Time reversal property). Suppose that the assumption of Theorem 2.3 is satisfied. Then

$$
\boldsymbol{n}\left(\Gamma^{\vee}\right)=\boldsymbol{n}(\Gamma), \quad \Gamma \in \mathcal{E}^{0} .
$$

\section{§2.5. Generalized Williams description}

Throughout this section, we suppose that the assumption of Theorem 2.3 is satisfied, i.e., we suppose that $m \in \mathcal{M}$ with $l=\infty$ and that the condition (S) is satisfied. For the symbols $\boldsymbol{P}^{x}$ and $\boldsymbol{Q}^{x}$, see $\S 4.1$ and $\S 4.2$ below, respectively.

Denote

$$
M(e)=\max _{t \geq 0} e(t), \quad e \in E .
$$

We prove the following in $\S 7.3$.

Lemma 2.1. It holds that

$$
\boldsymbol{n}(M \in d a)=\frac{d a}{a^{2}} \quad \text { on }(0, \infty) .
$$

Let $\left(Y^{1}(t): t \geq 0\right)$ and $\left(Y^{2}(t): t \geq 0\right)$ be two independent processes both of which obey the law $\boldsymbol{P}^{0}$. For $a \in(0, \infty)$, define

$$
Z^{a}(t)= \begin{cases}Y^{1}(t), & \text { if } 0 \leq t \leq \tau_{a}\left(Y^{1}\right), \\ Y^{2}\left(\tau_{a}\left(Y^{1}\right)+\tau_{a}\left(Y^{2}\right)-t\right), & \text { if } \tau_{a}\left(Y^{1}\right)<t \leq \tau_{a}\left(Y^{1}\right)+\tau_{a}\left(Y^{2}\right), \\ 0 & \text { if } t>\tau_{a}\left(Y^{1}\right)+\tau_{a}\left(Y^{2}\right) .\end{cases}
$$


Here $\tau_{a}$ denotes the first-entrance time to $[a, \infty)$ defined in (5.14). Set

$$
\boldsymbol{R}^{a}=\text { the law of }\left(Z^{a}(t): t \geq 0\right) \text { on the space } E^{0} .
$$

Now we state the generalized Williams description for our excursion measure $\boldsymbol{n}$.

Theorem 2.4. $\quad$ Suppose that the assumption of Theorem 2.3 is satisfied. Then

$$
\boldsymbol{n}(\Gamma)=\int_{0}^{\infty} \boldsymbol{R}^{a}(\Gamma) \frac{d a}{a^{2}}, \quad \Gamma \in \mathcal{E}
$$

The proof of Theorem 2.4 will be given in $\S 7.3$. It is based on two theorems.

The first one is the strong Markov property of the process $(e(t): t \geq 0)$ under $\boldsymbol{n}$. Let $\mathcal{E}_{(0, \tau)}$ and $\mathcal{E}_{(\tau, \zeta)}$ be $\sigma$-fields which represents the information of the path before and after the time $\tau$, respectively. Let $\mathfrak{X}_{\tau}^{+}$be the time-shift operator. Their precise definitions will be given in $\S 5.1$.

Theorem 2.5 (strong Markov property). Suppose that the assumption of Theorem 2.3 is satisfied. Let $\tau: E \rightarrow(0, \infty]$ be a positive stopping time which satisfies the assumption of Lemma 5.1 (iv). Then, for any $\Gamma_{1} \in \mathcal{E}_{(0, \tau)}$ and $\Gamma \in \mathcal{E}_{(\tau, \zeta)}$, it holds that

$$
\boldsymbol{n}\left(\Gamma_{1} \cap \Gamma\right)=\boldsymbol{P}^{0}\left[\mathbf{1}_{\Gamma_{1}}(w) \cdot \frac{1}{w(\tau)} \cdot \boldsymbol{Q}^{w(\tau)}\left(\mathfrak{X}_{\tau}^{+}(\Gamma)\right)\right] .
$$

Let $a \in(0, \infty)$ be fixed. We consider the first-entrance time $\tau_{a}$. Then we obtain the following.

Corollary 2.3. $\quad$ Suppose that the assumption of Theorem 2.3 is satisfied. Let $\Gamma_{1} \in \mathcal{E}_{\left(0, \tau_{a}\right)}$ and $\Gamma \in \mathcal{E}_{\left(\tau_{a}, \zeta\right)}$. Then it holds that

$$
\boldsymbol{n}\left(\Gamma_{1} \cap \Gamma\right)=\frac{1}{a} \boldsymbol{P}^{0}\left(\Gamma_{1}\right) \boldsymbol{Q}^{a}\left(\mathfrak{X}_{\tau_{a}}^{+}(\Gamma)\right) .
$$

This is an immediate consequence of Theorem 2.5 so that we omit the proof.

From this corollary, the following is derived.

Corollary 2.4. Suppose that the assumption of Theorem 2.3 is satisfied. For $a \in(0, \infty)$, it holds that

$$
\boldsymbol{n}\left[e^{-\lambda \tau_{a}}\right]=\frac{1}{\psi_{\lambda}(a)} .
$$


In particular, it holds that

$$
\boldsymbol{n}\left(\tau_{a}<\infty\right)=\frac{1}{a}
$$

Hence the measure $\boldsymbol{n}_{a}=$ an $\left.\right|_{E^{\tau_{a}}}$ defines a probability measure on $\left(E^{\tau_{a}}, \mathcal{E}^{\tau_{a}}\right)$.

The proofs of Theorem 2.5 and Corollary 2.4 will be given in $\S 7.1$.

The second one is the first-entrance-last-exit decomposition. This formula unifies the first-entrance decomposition (see e.g. [16]) and the last-exit one (see e.g. [16] and [17]) in a single framework.

Let $a \in(0, \infty)$ be fixed. Let $\epsilon_{a}$ denote the last-exit time from $[a, \infty)$, which will be introduced in $\S 5.2$. Let $\mathcal{E}_{\left(0, \tau_{a}\right)}^{0, a}, \mathcal{E}_{\left(\tau_{a}, \epsilon_{a}\right)}^{0, a}$ and $\mathcal{E}_{\left(\epsilon_{a}, \zeta\right)}^{0, a}$ be $\sigma$-fields which represent the information of the path on the intervals indicated in the subscripts. These precise definitions will be given in $\S 5.3$.

Theorem 2.6 (The first-entrance-last-exit decomposition). Suppose that the assumption of Theorem 2.3 is satisfied. Let $\Gamma_{1} \in \mathcal{E}_{\left(0, \tau_{a}\right)}^{0, a}, \Gamma_{2} \in \mathcal{E}_{\left(\tau_{a}, \epsilon_{a}\right)}^{0, a}$ and $\Gamma_{3} \in \mathcal{E}_{\left(\epsilon_{a}, \zeta\right)}^{0, a}$. Then the following decomposition holds:

$$
\boldsymbol{n}\left(\Gamma_{1} \cap \Gamma_{2} \cap \Gamma_{3}\right)=\frac{1}{a} \boldsymbol{P}^{0}\left(\Gamma_{1}\right) \cdot \boldsymbol{Q}^{a}\left(\mathfrak{X}_{\tau_{a}}^{+}\left(\Gamma_{2}\right)\right) \cdot \boldsymbol{P}^{0}\left(\left(\Gamma_{3}\right)^{\vee}\right) .
$$

The proof will be given in $\S 7.2$.

Noting that the lifetime interval is divided into three pieces as

$$
[0, \zeta]=\left[0, \tau_{a}\right] \cup\left(\tau_{a}, \epsilon_{a}\right) \cup\left[\epsilon_{a}, \zeta\right]
$$

we have the following.

Corollary 2.5. $\quad$ Suppose that the assumption of Theorem 2.3 is satisfied. Then the joint distribution of the length of the three intervals $\left[0, \tau_{a}\right],\left(\tau_{a}, \epsilon_{a}\right)$ and $\left[\epsilon_{a}, \zeta\right]$ is given by

$$
\begin{aligned}
& \boldsymbol{n}\left(\left\{\tau_{a} \in d t_{1}\right\} \cap\left\{\epsilon_{a}-\tau_{a} \in d t_{2}\right\} \cap\left\{\zeta-\epsilon_{a} \in d t_{3}\right\}\right) \\
= & \frac{1}{a} \boldsymbol{P}^{0}\left(\tau_{a} \in d t_{1}\right) \boldsymbol{Q}^{a}\left(\epsilon_{a} \in d t_{2}\right) \boldsymbol{P}^{0}\left(\tau_{a} \in d t_{3}\right) .
\end{aligned}
$$

The proof is obvious and is omitted. 


\section{$\S 2.6$. Convergence theorem of integrals with respect to Poisson point fields}

Let $m \in \mathcal{M}$ with $l=\infty$ be such that the condition $\left(\mathbf{S}^{*}\right)$ is satisfied. Then Theorem 2.2 is valid and thus $(\mathbf{S})$ is also satisfied.

We denote the $\boldsymbol{n}, \rho$ and $\sigma^{*}$ for $m$ by $\boldsymbol{n}(m ; \cdot), \rho(m ; \cdot)$ and $\sigma^{*}(m ; \cdot)$, respectively.

Since the measure $\boldsymbol{n}(m ; \cdot)$ is $\sigma$-finite, there corresponds a Poisson point field $\boldsymbol{N}(m ; d t, d e)$ on the space $(0, \infty) \times E^{0}$ with intensity measure $d t \boldsymbol{n}(m ; d e)$ on a probability space $(\Omega, \mathcal{F}, \boldsymbol{P})$. That is,

$$
\boldsymbol{P}\left[\exp \left(-\int_{E^{0}} F(e) \boldsymbol{N}(m ;(0, t], d e)\right)\right]=\exp \left(-t \int_{E^{0}}\left(1-e^{-F(e)}\right) \boldsymbol{n}(m ; d e)\right)
$$

for any $t \geq 0$ and any non-negative measurable function $F$ on $E^{0}$. Define a filtration

$$
\mathcal{F}_{t}=\sigma\left\{\boldsymbol{N}(m ;(s, t], \Gamma): 0<s<t<\infty, \Gamma \in \mathcal{E}^{0}\right\}, \quad t \geq 0
$$

and define a random measure $\widetilde{\boldsymbol{N}}(m ; d t, d e)$ by

$$
\widetilde{\boldsymbol{N}}(m ; d t, d e)=\boldsymbol{N}(m ; d t, d e)-d t \boldsymbol{n}(m ; d e) .
$$

Then, for any measurable function $F$ on $E^{0}$ such that

$$
\int_{E^{0}}|F(e)|^{2} \boldsymbol{n}(m ; d e)<\infty
$$

the process

$$
M[F](t)=\int_{E^{0}} F(e) \widetilde{\boldsymbol{N}}(m ;(0, t], d e), \quad t \geq 0
$$

is a square-integrable $\left(\mathcal{F}_{t}\right)$-martingale with quadratic variation

$$
\langle M[F]\rangle_{t}=t \int_{E^{0}}|F(e)|^{2} \boldsymbol{n}(m ; d e), \quad t \geq 0
$$

and each of whose increments $M[F](t)-M[F](s)$ is independent of $\mathcal{F}_{s}$ for $0 \leq s<t$.

In the sequel, we assume that $m \in \mathcal{M}_{1}$ with $l=\infty$. Then the conditions (S) and $\left(\mathbf{S}^{*}\right)$ are satisfied. 
For a function $f$ on $(0, \infty)$, we want to define the integrals

$$
U_{1}[f](m ; t)=\int_{\{\zeta<1\}} f(\zeta(e)) \widetilde{\boldsymbol{N}}(m ;(0, t], d e), \quad t \geq 0
$$

and

$$
U_{2}[f](m ; t)=\int_{\{\zeta \geq 1\}} f(\zeta(e)) \boldsymbol{N}(m ;(0, t], d e), \quad t \geq 0 .
$$

The following lemma gives a sufficient condition on $f$ for the integrals (2.53) and (2.54) to be well-defined.

Lemma 2.2. Suppose that $m \in \mathcal{M}_{1}$ with $l=\infty$.

(i) Let $f$ be a measurable function on $(0,1)$ such that

$$
|f(u)| \leq C u, \quad 0<u<1
$$

for some constant $C$. Then it holds that

$$
\int_{0}^{1}|f(u)|^{2} \rho(m ; u) d u<\infty .
$$

Hence the stochastic integral $U_{1}[f](m ; t)$ given in $(2.53)$ is well-defined.

(ii) It holds that

$$
\boldsymbol{P}(\boldsymbol{N}(m ;(0, t],\{\zeta \geq 1\})<\infty)=1, \quad t \geq 0 .
$$

Hence, for any measurable function $f$ on $[1, \infty)$, the integral $U_{2}[f](m ; t)$ given in (2.54) is well-defined (as a finite sum).

(iii) If both of the assumptions of (i) and (ii) are satisfied, then the processes $\left(U_{1}[f](m ; t)\right)$ and $\left(U_{2}[f](m ; t)\right)$ are independent.

The proof will be given in $\S 8$.

Suppose that the assumption of Lemma 2.2 (i) is satisfied. Then the process $\left(U_{1}[f](m ; t)\right)$ defined by $(2.53)$ is a square-integrable $\left(\mathcal{F}_{t}\right)$-martingale with quadratic variation

$$
\begin{aligned}
\left.\left\langle U_{1}[f](m ;)\right)\right\rangle_{t} & =t \int_{\{\zeta<1\}}|f(\zeta(e))|^{2} \boldsymbol{n}(m ; d e) \\
& =t \int_{0}^{1}|f(u)|^{2} \rho(m ; u) d u
\end{aligned}
$$

(here we used Corollary 2.1) and each of whose increments $U_{1}[f](m ; t)-U_{1}[f]$ $(m ; s)$ is independent of $\mathcal{F}_{s}$ for $0<s<t$.

The following theorem assures the continuity of the maps $m \mapsto U_{1}[f](m ; t)$ and $U_{2}[f](m ; t)$. 
Theorem 2.7. $\quad$ Suppose that $m_{n}, m \in \mathcal{M}_{1}$ with $l\left(m_{n}\right)=l(m)=\infty$ and that $m_{n} \rightarrow m$ in $\mathcal{M}_{1}$.

(i) Suppose that the assumption of Lemma 2.2 (i) is satisfied. Then

$$
U_{1}[f]\left(m_{n} ; t\right) \stackrel{\text { law }}{\longrightarrow} U_{1}[f](m ; t) \quad \text { as } n \rightarrow \infty, \quad t \geq 0 .
$$

(ii) Let $f$ be a measurable function on $[1, \infty)$ such that $\lim _{u \rightarrow \infty} f(u)=c$ for some $c \in[-\infty, \infty]$. Then

$$
U_{2}[f]\left(m_{n} ; t\right) \stackrel{\text { law }}{\longrightarrow} U_{2}[f](m ; t) \quad \text { as } n \rightarrow \infty, \quad t \geq 0 .
$$

The proof will be given in $\S 8$.

Recall Theorem 2.1 of Kasahara-Watanabe [8], stated in $\S 2.1$. The following corollary generalizes Theorem 2.1 in terms of the Poisson point fields.

Corollary 2.6. Suppose that the assumption of Theorem 2.1 is satisfied. Suppose, moreover, that $f$ satisfies all the assumptions of Theorem 2.7. Set

$$
f_{\lambda}(x)=f\left(\frac{1}{\lambda^{1 / \alpha} L(\lambda)} x\right)
$$

Then it holds that

$$
U_{1}\left[f_{\lambda}\right](m ; \lambda t) \stackrel{\text { law }}{\longrightarrow} U_{1}[f]\left(m^{(\alpha)} ; t\right)
$$

and

$$
U_{2}\left[f_{\lambda}\right](m ; \lambda t) \stackrel{\text { law }}{\longrightarrow} U_{2}[f]\left(m^{(\alpha)} ; t\right)
$$

as $\lambda \rightarrow \infty$ for $t \geq 0$.

The proof will be given in $\S 8$.

\section{$\S 3 . \quad$ Notations and Preliminaries (I): The Fundamental Solutions and Spectral Measures}

\section{§3.1. The fundamental solution of $L_{m}$}

Let $m \in \mathcal{M}$ and consider the operator $L_{m}$ on $\mathcal{D}\left(L_{m}\right)$ introduced in $\S 2.3$.

For $\lambda \in \mathbb{C}$, we denote by $\psi_{\lambda}$ the unique solution of the integral equation

$$
\psi_{\lambda}(x)=x+\lambda \int_{(0, x]}(x-y) \psi_{\lambda}(y) d m(y) \quad \text { on }[0, l) .
$$


For $\lambda>0$, this is equivalent to say that $u=\psi_{\lambda}$ is the unique increasing solution of $\mathcal{L}_{m} u=\lambda u$ with initial condition

$$
\psi_{\lambda}(0)=0, \quad \psi_{\lambda}^{\prime}(0)=1 .
$$

For fixed $x \in[0, l)$, the function $\lambda \mapsto \psi_{\lambda}(x)$ is an entire function on $\mathbb{C}$.

For $\lambda>0$, we define

$$
g_{\lambda}(x)=\psi_{\lambda}(x) \int_{x}^{l} \frac{d y}{\psi_{\lambda}(y)^{2}}, \quad x \in(0, l)
$$

so that the Wronskian is given by

$$
\psi_{\lambda}^{\prime}(x) g_{\lambda}(x)-\psi_{\lambda}(x) g_{\lambda}^{\prime}(x)=1, \quad x \in(0, l) .
$$

Then $u=g_{\lambda}$ is the unique decreasing solution of $\mathcal{L}_{m} u=\lambda u$ such that

$$
g_{\lambda}(0+)=1
$$

and

$$
\begin{cases}g_{\lambda}(l-)=0 & \text { if } x=l \text { is exit, } \\ g_{\lambda}^{\prime}(l-)=0 & \text { if } x=l \text { is entrance and non-regular. }\end{cases}
$$

The resolvent operator $\left(\lambda-L_{m}\right)^{-1}$ has a continuous kernel given by

$$
G(\lambda, x, y)=G(\lambda, y, x)=g_{\lambda}(x) \psi_{\lambda}(y), \quad \lambda>0,0<x \leq y<l .
$$

It is known that there exists a non-negative Radon measure $\theta$ on $(0, \infty)$, which is called the spectral measure, such that

$$
G(\lambda, x, y)=\int_{(0, \infty)} \frac{\psi_{-\xi}(x) \psi_{-\xi}(y)}{\lambda+\xi} \theta(d \xi), \quad \lambda>0, x, y \in(0, l) .
$$

We remark that the spectral measure $\theta$ does not have a point mass at $\xi=0$, since $\psi_{0}(x)=x$ never belongs to $\mathcal{D}\left(L_{m}\right)$. Letting $x=y \in(0, l)$, we have

$$
G(\lambda, x, x)=\int_{(0, \infty)} \frac{\left|\psi_{-\xi}(x)\right|^{2}}{\lambda+\xi} \theta(d \xi)<\infty, \quad \lambda>0, x \in(0, l),
$$

and hence the integral in the RHS of (3.8) converges absolutely. In addition, the expression

$$
q(t, x, y)=\int_{(0, \infty)} e^{-t \xi} \psi_{-\xi}(x) \psi_{-\xi}(y) \theta(d \xi), \quad t>0, x, y \in(0, l)
$$


gives the eigendifferential expansion of the fundamental solution of $L_{m}$. It is obvious that

$$
G(\lambda, x, y)=\int_{0}^{\infty} e^{-\lambda t} q(t, x, y) d t, \quad \lambda>0, x, y \in(0, l) .
$$

\section{§3.2. Proof of Proposition 2.1}

For the proof of Proposition 2.1, we prepare the following.

Lemma 3.1. Suppose that the assumption (S) is satisfied. Then, for any $t>0$, there exists $a(t) \in(0, l)$ such that

$$
\int_{(0, \infty)} e^{-t \xi}\left(\sup _{x \in(0, a(t)]}\left|\psi_{-\xi}^{\prime}(x)\right|^{2}\right) \theta(d \xi)<\infty
$$

Proof of Lemma 3.1. Let $\delta>0$ be fixed. Set

$$
F(a)=\int_{(0, a]}\left|\psi_{-\xi}(x)\right| d m(x), \quad \xi>0, a \in(0, \delta) .
$$

By the integral equation (3.1), we have

$$
F(a) \leq c(\delta)+\xi \int_{(0, a]} F(x) x d m(x), \quad \xi>0, a \in(0, \delta)
$$

for some $\delta>0$, where

$$
c(a)=\int_{(0, a]} x d m(x)<\infty
$$

by the assumption $m \in \mathcal{M}$. Then Gronwall's lemma says that

$$
F(a) \leq c(\delta) e^{\xi c(a)}, \quad \xi \geq 0, a \in(0, \delta) .
$$

By the integral equation (3.1) again, we have

$$
\psi_{-\xi}^{\prime}(x)=1-\xi \int_{(0, x]} \psi_{-\xi}(y) d m(y) .
$$

Using the inequality $(a+b)^{2} \leq 2\left(a^{2}+b^{2}\right)$ and the estimate (3.16), we have

$$
\sup _{x \in[0, a)}\left|\psi_{-\xi}^{\prime}(x)\right|^{2} \leq 2+2 \xi^{2} c(\delta)^{2} e^{2 \xi c(a)}, \quad \xi \geq 0, a \in(0, \delta) .
$$


Since $\lim _{a \rightarrow 0+} c(a)=0$, we can take $a(t)$ so that $2 c(a)<t$ for any $a \in(0, a(t))$. Therefore we obtain (3.12) by the assumption $(\mathbf{S})$.

Proof of Proposition 2.1. We only prove the claim (i), since (ii) and (iii) are similar as and easier than (i). Let $t>0$ and $x \in(0, l)$ be fixed and take $a(t)$ as in Lemma 3.1. Then we have

$$
\int_{(0, \infty)} e^{-t \xi}\left|\psi_{-\xi}(x)\right|\left(\sup _{y \in(0, a(t)]}\left|\psi_{-\xi}^{\prime}(y)\right|\right) \theta(d \xi)<\infty .
$$

Thus we can apply the dominated convergence to obtain

$$
\frac{\partial q}{\partial y}(t, x, y)=\int_{(0, \infty)} e^{-t \xi} \psi_{-\xi}(x) \psi_{-\xi}^{\prime}(y) \theta(d \xi), \quad y \in(0, a(t))
$$

where it is continuous in $y \in(0, a(t))$. Letting $y \rightarrow 0+$, we obtain

$$
\frac{\partial q}{\partial y}(t, x, 0+)=\int_{(0, \infty)} e^{-t \xi} \psi_{-\xi}(x) \theta(d \xi), \quad y \in(0, a(t)),
$$

since $\psi_{-\xi}^{\prime}(0+)=1$. Noting that $\psi_{-\xi}(0)=0$ and that $\psi_{-\xi}(x)=\int_{0}^{x} \psi_{-\xi}^{\prime}(y) d y$, we obtain (2.21) in a similar argument. The third expression of (2.21) implies that the function $\Pi(t, x)$ is non-negative.

\section{§3.3. Harmonic transform}

Let $m \in \mathcal{M}$. We consider the harmonic transform of $\mathcal{L}_{m}$ with respect to the harmonic function $\psi_{0}(x)=x$.

We define

$$
m^{h}(x)=\int_{(0, x]} y^{2} d m(y), \quad s^{h}(x)=-\frac{1}{x}, \quad x \in(0, l)
$$

and we consider the operator

$$
\mathcal{L}_{m}^{h}=\mathcal{L}_{\left(m^{h}, s^{h}\right)}=\frac{d}{d m^{h}} \frac{d}{d s^{h}}=\frac{1}{x^{2}} \frac{d}{d m}\left(x^{2} \frac{d}{d x}\right) .
$$

We define

$$
\mathcal{D}\left(L_{m}^{h}\right)=\left\{v(x)=\frac{u(x)}{x}: u \in \mathcal{D}\left(L_{m}\right)\right\}
$$

and

$$
L_{m}^{h} v=\frac{1}{x} L_{m}(x v), \quad v \in \mathcal{D}\left(L_{m}^{h}\right) .
$$

Then the operator $L_{m}^{h}$ on the domain $\mathcal{D}\left(L_{m}^{h}\right)$ is self-adjoint. 
Remark 5. By (3.22), we easily see that the boundary $x=0$ for $\mathcal{L}_{m}^{h}$ is entrance and non-regular in any case, but that $x=l$ for $\mathcal{L}_{m}^{h}$ is possibly regular. The boundary condition at $x=0$ is necessarily reflecting. If $x=l$ is regular, we adopt the reflecting boundary condition at $x=l$ by choosing the domain $\mathcal{D}\left(L_{m}^{h}\right)$ as above.

We define

$$
\phi_{\lambda}^{h}(x)=\frac{\psi_{\lambda}(x)}{x}, \quad \lambda \in \mathbb{C}, x \in(0, l)
$$

and

$$
f_{\lambda}^{h}(x)=\frac{g_{\lambda}(x)}{x}, \quad \lambda>0, x \in(0, l) .
$$

Then, for $\lambda \in \mathbb{C}$, the function $\phi_{\lambda}^{h}(x)$ is the unique solution of the equation

$$
\phi_{\lambda}^{h}(x)=1+\lambda \int_{(0, x]}(x-y) \phi_{\lambda}^{h}(y) d m^{h}(y) \quad \text { on }[0, l) .
$$

In addition, for $\lambda>0$, the function $u(x)=\phi_{\lambda}^{h}(x)$ is the unique positive increasing solution of $\mathcal{L}_{m}^{h} u=\lambda u$ with initial condition

$$
\phi_{\lambda}^{h}(0+)=1, \quad \frac{d \phi_{\lambda}^{h}}{d s^{h}}(0+)=0 .
$$

For $\lambda>0$, the function $u=f_{\lambda}^{h}(x)$ is a positive decreasing solution of $\mathcal{L}_{m}^{h} u=\lambda u$ which satisfies

$$
f_{\lambda}^{h}(x)=\phi_{\lambda}^{h}(x) \int_{(x, l)} \frac{d s^{h}(y)}{\phi_{\lambda}^{h}(y)^{2}}, \quad x \in(0, l)
$$

and

$$
\frac{d \phi_{\lambda}^{h}}{d s^{h}}(x) f_{\lambda}^{h}(x)-\phi_{\lambda}^{h}(x) \frac{d f_{\lambda}^{h}}{d s^{h}}(x)=1, \quad \lambda>0, x \in(0, l) .
$$

Define

$$
p^{h}(t, x, y)=\frac{q(t, x, y)}{x y}, \quad t>0, x, y \in(0, l) .
$$

Then the resolvent kernel of $\mathcal{L}_{m}^{h}$ is given by

$$
G^{h}(\lambda, x, y)=\frac{G(\lambda, x, y)}{x y}=\int_{(0, \infty)} \frac{\phi_{-\xi}^{h}(x) \phi_{-\xi}^{h}(y)}{\lambda+\xi} \theta(d \xi)
$$


and the fundamental solution of $\mathcal{L}_{m}^{h}$ is given by

$$
p^{h}(t, x, y)=\frac{q(t, x, y)}{x y}=\int_{(0, \infty)} e^{-t \xi} \phi_{-\xi}^{h}(x) \phi_{-\xi}^{h}(y) \theta(d \xi) .
$$

It is obvious that

$$
p^{h}(t, x, 0+)=p^{h}(t, 0+, x)=\frac{\Pi(t, x)}{x}, \quad t>0, x \in(0, l)
$$

and that

$$
p^{h}(t, 0+, 0+)=\rho(t), \quad t>0 .
$$

\section{§3.4. Dual string}

Let $m \in \mathcal{M}$. In order to study the operator $\mathcal{L}_{m^{*}}$ for the dual string $m^{*}(x)=m^{-1}(x)$, we define

$$
m^{d}(x)=x, \quad s^{d}(x)=m(x), \quad x \in(0, l)
$$

and consider the operator

$$
\mathcal{L}_{m}^{d}=\frac{d}{d m^{d}} \frac{d}{d s^{d}} .
$$

Then its scale transform $x^{\prime}=s^{d}(x)=m(x)$ of the operator $\mathcal{L}_{m}^{d}$ coincides with the operator $\mathcal{L}_{m^{*}}$.

Define

$$
\phi_{\lambda}^{d}(x)=\psi_{\lambda}^{\prime}(x), \quad \lambda \in \mathbb{C}, x \in[0, l)
$$

and

$$
f_{\lambda}^{d}(x)=-\frac{1}{\lambda} g_{\lambda}^{\prime}(x), \quad \lambda>0, x \in(0, l) .
$$

Then the function $\phi_{\lambda}^{d}$ is the unique solution of the equation

$$
\phi_{\lambda}^{d}(x)=1+\lambda \int_{(0, x]}\left(s^{d}(x)-s^{d}(y)\right) \phi_{\lambda}^{d}(y) d m^{d}(y), \quad \lambda \in \mathbb{C}, x \in[0, l) .
$$

For $\lambda>0$, the function $f_{\lambda}^{d}$ satisfies

$$
f_{\lambda}^{d}(x)=\phi_{\lambda}^{d}(x) \int_{x}^{l} \frac{d s^{d}(y)}{\phi_{\lambda}^{d}(y)^{2}}, \quad \lambda>0, x \in(0, l),
$$


since

$$
\left(\phi_{\lambda}^{d}\right)^{\prime}(x) f_{\lambda}^{d}(x)-\phi_{\lambda}^{d}(x)\left(f_{\lambda}^{d}\right)^{\prime}(x)=1, \quad \lambda>0, x \in(0, l) .
$$

In addition, $f_{\lambda}^{d}$ is the unique decreasing solution of $\mathcal{L}_{m}^{d}$ such that

$$
\left(f_{\lambda}^{d}\right)^{\prime}(0+)=-1
$$

and

$$
\begin{cases}\left(f_{\lambda}^{d}\right)^{\prime}(l-)=0 & \text { if } x=l \text { for } \mathcal{L}_{m} \text { is exit, } \\ f_{\lambda}^{d}(l-)=0 & \text { if } x=l \text { for } \mathcal{L}_{m} \text { is entrance and non-regular. }\end{cases}
$$

Keeping (3.41) and (3.45) in mind, we adopt the reflecting boundary condition at $x=0$, and adopt the reflecting or absorbing condition at $x=l$ according as $x=l$ for $\mathcal{L}_{m}$ is exit or entrance and non-regular. Under these conditions, we denote the unique self-adjoint extension of $\mathcal{L}_{m}^{d}$ by $L_{m}^{d}$ with its domain $\mathcal{D}\left(L_{m}^{d}\right)$.

There exists a non-negative Radon measure $\sigma^{*}$ on $[0, \infty)$ such that

$$
G^{d}(\lambda, x, y)=\int_{[0, \infty)} \frac{\phi_{-\xi}^{d}(x) \phi_{-\xi}^{d}(y)}{\lambda+\xi} \sigma^{*}(d \xi), \quad \lambda>0, x, y \in(0, l)
$$

and

$$
p^{d}(t, x, y)=\int_{[0, \infty)} e^{-t \xi} \phi_{-\xi}^{d}(x) \phi_{-\xi}^{d}(y) \sigma^{*}(d \xi), \quad t>0, x, y \in(0, l),
$$

where $G^{d}(\lambda, x, y)$ and $p^{d}(t, x, y)$ are the resolvent kernel and the fundamental solution of $L_{m}^{d}$, respectively. Moreover, it holds that

$$
\sigma^{*}(\{0\})=\frac{1}{l} .
$$

Now we are in a position to prove Theorem 2.2.

Proof of Theorem 2.2. Note that

$$
G^{d}(\lambda, x, y)=\phi_{\lambda}^{d}(x) f_{\lambda}^{d}(y)=-\frac{1}{\lambda} \psi_{\lambda}^{\prime}(x) g_{\lambda}^{\prime}(y), \quad \lambda>0,0<x<y<l .
$$

Since $\psi_{\lambda}(0)=0$, we have

$$
\int_{0}^{x} d u \int_{y_{1}}^{y_{2}} d v G^{d}(\lambda, u, v)=\frac{1}{\lambda} \psi_{\lambda}(x)\left(g_{\lambda}\left(y_{1}\right)-g_{\lambda}\left(y_{2}\right)\right)
$$


for $\lambda>0$ and $0<x<y_{1}<y_{2}<l$. Note that

$$
\begin{aligned}
\frac{1}{\lambda} \psi_{\lambda}(x) g_{\lambda}(y) & =\frac{x y}{\lambda} \phi_{\lambda}^{h}(x) f_{\lambda}^{h}(y) \\
& =\frac{x y}{\lambda} \int_{0}^{\infty} e^{-\lambda t} p^{h}(t, x, y) d t \\
& =x y \int_{0}^{\infty} e^{-\lambda t} d t \int_{0}^{t} p^{h}(s, x, y) d s .
\end{aligned}
$$

Then we can rewrite $(3.50)$ as

$$
\begin{aligned}
& \int_{0}^{\infty} e^{-\lambda t} d t\left\{\int_{0}^{x} d u \int_{y_{1}}^{y_{2}} d v p^{d}(t, u, v)+\int_{0}^{t} x y_{2} p^{h}\left(s, x, y_{2}\right) d s\right\} \\
& =\int_{0}^{\infty} e^{-\lambda t} d t \int_{0}^{t} x y_{1} p^{h}\left(s, x, y_{1}\right) d s .
\end{aligned}
$$

Taking Laplace inversion, we have

$$
\int_{0}^{x} d u \int_{y_{1}}^{y_{2}} d v p^{d}(t, u, v)=\int_{0}^{t} d s\left\{x y_{1} p^{h}\left(s, x, y_{1}\right)-x y_{2} p^{h}\left(s, x, y_{2}\right)\right\}
$$

Let $t_{0}>0$ be fixed. Under the assumption $\left(\mathbf{S}^{*}\right)$, the integral

$$
\int_{(0, \infty)} e^{-t \xi} \phi_{-\xi}^{d}(x) \phi_{-\xi}^{d}(y) \xi \sigma^{*}(d \xi)
$$

converges absolutely and uniformly in $x, y \in\left(0, a\left(t_{0}\right)\right)$ for any $t>t_{0}$. Thus the function $p^{d}(t, u, v)$ is differentiable with respect to $t$ and its derivative is continuous in $(u, v)$ on $\left(0, a\left(t_{0}\right)\right) \times\left(0, a\left(t_{0}\right)\right)$.

Differentiating both sides of $(3.56)$ with respect to $t$, we have

$$
\int_{0}^{x} d u \int_{y_{1}}^{y_{2}} d v \frac{\partial p^{d}}{\partial t}(t, u, v)=x y_{1} p^{h}\left(t, x, y_{1}\right)-x y_{2} p^{h}\left(t, x, y_{2}\right) .
$$

Taking $y_{1}=x$ and $y_{2}=x+h$ with $h>0$, dividing both sides by $-h x$ and letting $h \rightarrow 0+$, we have

$$
-\frac{1}{x} \int_{0}^{x} d u \frac{\partial p^{d}}{\partial t}(t, u, x)=p^{h}(t, x, x) .
$$

Since the LHS converges, the $\operatorname{limit}_{x \rightarrow 0+} \lim ^{h}(t, x, x)$ exists and we obtain

$$
\lim _{x \rightarrow 0+} p^{h}(t, x, x)=-\frac{\partial p^{d}}{\partial t}(t, 0+, 0+)
$$


We apply Fatou's lemma to obtain

$\int_{(0, \infty)} e^{-t \xi} \theta(d \xi) \leq \liminf _{x \rightarrow 0+} \int_{(0, \infty)} e^{-t \xi}\left(\frac{\psi_{-\xi}(x)}{x}\right)^{2} \theta(d \xi)=\lim _{x \rightarrow 0+} p^{h}(t, x, x)<\infty$,

which proves $(\mathbf{S})$. Therefore we combine (3.36) and (3.60) to obtain

$$
\int_{(0, \infty)} e^{-t \xi} \xi \sigma^{*}(d \xi)=\int_{(0, \infty)} e^{-t \xi} \theta(d \xi)
$$

which completes the proof.

\section{§3.5. Strings of limit circle type}

In this subsection, we always assume $m \in \mathcal{M}$ and denote the $\sigma^{*}$ for $m \in \mathcal{M}$ by $\sigma^{*}(m ; \cdot)$.

It is well-known (see, e.g., [7] and [13]) that $m \in \mathcal{M}_{0}$ if and only if

$$
\int_{[0, \infty)} \frac{\sigma^{*}(m ; d \xi)}{1+\xi}<\infty
$$

and that $m_{n}(x) \rightarrow m(x)$ at every continuity point $x$ of $m$ if and only if

$$
\int_{[0, \infty)} \frac{\sigma^{*}\left(m_{n} ; d \xi\right)}{\lambda+\xi} \rightarrow \int_{[0, \infty)} \frac{\sigma^{*}(m ; d \xi)}{\lambda+\xi}, \quad \lambda>0
$$

Kotani [12] and Kasahara-Watanabe [8] have studied a generalization of the above result.

Definition 3.1. Let $m_{n}, m \in \mathcal{M}_{1}$. It is said that $m_{n} \rightarrow m$ in $\mathcal{M}_{1}$ if the following two conditions hold:

(i) $m_{n}(x) \rightarrow m(x)$ at every continuity point $x$ of $m$.

(ii) $\lim _{\delta \rightarrow 0+} \limsup _{n \rightarrow \infty} \int_{0}^{\delta} m_{n}(x)^{2} d x=0$.

Then the following holds.

Theorem 3.1 (Kasahara-Watanabe [8], Kotani [12]). $\quad$ Let $m \in \mathcal{M}$.

(i) $m \in \mathcal{M}_{1}$ if and only if

$$
\int_{[0, \infty)} \frac{\sigma^{*}(m ; d \xi)}{1+\xi^{2}}<\infty
$$


(ii) If $m_{n} \in \mathcal{M}_{1}$ converges to $m \in \mathcal{M}_{1}$ in $\mathcal{M}_{1}$, then it holds that

$$
h\left(m_{n} ; \lambda\right) \rightarrow h(m ; \lambda), \quad \lambda>0 .
$$

Here

$$
h(m ; \lambda)=\int_{[0, \infty)}\left(\frac{1}{\lambda+\xi}-\frac{\xi}{1+\xi^{2}}\right) \sigma^{*}(m ; d \xi) .
$$

Remark 6. If $m \in \mathcal{M}_{1}$, then (i) implies that $\left(\mathbf{S}^{*}\right)$ is satisfied and hence Theorem 2.2 is valid. Thus (S) is also satisfied and hence Proposition 2.1 is also valid.

For later use, we prepare the following.

Lemma 3.2. If $m_{n} \rightarrow m$ in $\mathcal{M}_{1}$, then

$$
\int_{[0, \infty)} F(\xi) \frac{\sigma^{*}\left(m_{n} ; d \xi\right)}{1+\xi^{2}} \rightarrow \int_{[0, \infty)} F(\xi) \frac{\sigma^{*}(m ; d \xi)}{1+\xi^{2}}
$$

for any bounded continuous function $F$ on $[0, \infty)$.

Proof. Suppose that $m_{n} \rightarrow m$ in $\mathcal{M}_{1}$. Then, by (ii) of Theorem 3.1, we have

$$
\int_{[0, \infty)} \frac{\sigma^{*}\left(m_{n} ; d \xi\right)}{(\lambda+2+\xi)(1+\xi)} \rightarrow \int_{[0, \infty)} \frac{\sigma^{*}(m ; d \xi)}{(\lambda+2+\xi)(1+\xi)}
$$

for $\lambda>-1$. Let

$$
\mu(m ; d \xi)=\left(\int_{[0, \infty)} \frac{\sigma^{*}(m ; d \xi)}{(2+\xi)(1+\xi)}\right)^{-1} \frac{\sigma^{*}(m ; d \xi)}{(2+\xi)(1+\xi)} .
$$

Then $\mu(m ; d \xi)$ is a probability measure and we have

$$
\int_{[0, \infty)} \frac{2+\xi}{\lambda+2+\xi} \mu\left(m_{n} ; d \xi\right) \rightarrow \int_{[0, \infty)} \frac{2+\xi}{\lambda+2+\xi} \mu(m ; d \xi), \quad \lambda>-1 .
$$

We rewrite the integral as

$$
\begin{aligned}
\int_{[0, \infty)} \frac{2+\xi}{\lambda+2+\xi} \mu(m ; d \xi) & =\int_{0}^{\infty} e^{-\lambda t} d t \int_{[0, \infty)}(2+\xi) e^{-(2+\xi) t} \mu(m ; d \xi) \\
& =\lambda \int_{0}^{\infty} e^{-\lambda s} d s \int_{[0, \infty)}\left(1-e^{-(2+\xi) s}\right) \mu(m ; d \xi)
\end{aligned}
$$


for $\lambda>-1$. Thus we apply the continuity theorem of Laplace transform to obtain

$$
\int_{[0, \infty)} e^{-s(2+\xi)} \mu\left(m_{n} ; d \xi\right) \rightarrow \int_{[0, \infty)} e^{-s(2+\xi)} \mu(m ; d \xi), \quad s>0 .
$$

We apply the continuity theorem again to obtain the desired result.

\section{§4. Notations and Preliminaries (II): Diffusion Processes}

For a detail treatment of what is developed in this section, see, e.g., [6], [4] and [15].

\section{$\S 4.1 . \quad L_{m}^{h}$-diffusion process}

Let $W$ be the totality of continuous paths on $[0, \infty)$ :

$$
W=\{w:[0, \infty) \rightarrow[0, \infty): \text { continuous }\}
$$

Denote by $\mathcal{W}$ the $\sigma$-field generated by cylinder sets of the form

$$
V=\left\{w \in W: w\left(t_{1}\right) \in A_{1}, \ldots, w\left(t_{n}\right) \in A_{n}\right\}
$$

for some $0=t_{0}<t_{1}<\cdots<t_{n}<\infty$ and $A_{1}, \ldots, A_{n} \in \mathcal{B}([0, \infty))$. For $0<t<\infty$, we denote by $\mathcal{W}_{t}$ the $\sigma$-field generated by such cylinder functions $V$ of the form (4.2) where $0<t_{1}<\cdots<t_{n} \leq t$.

Then we can construct a family of probability measures $\left(\boldsymbol{P}^{x}: x \in[0, l)\right)$ on $W$ under which the coordinate process is a generalized $L_{m}^{h}$-diffusion with $x=l$ a trap if $x=l$ is absorbing, i.e., the Markovian family with the transition probability given by $p^{h}(t, x, y) d m^{h}(y)$.

Let $\boldsymbol{P}_{t}^{x, y}$ for $x, y \in[0, l)$ denote the conditional law of the $L_{m}^{h}$-diffusion process starting from $x$ conditioned on $w(t)=y$ :

$$
\boldsymbol{P}_{t}^{x, y}(\Gamma)=\boldsymbol{P}^{x}(\Gamma \mid w(t)=y), \quad \Gamma \in \mathcal{W} .
$$

\section{$\S 4.2 . \quad L_{m}$-diffusion process}

We can also construct a family of probability measures $\left(\boldsymbol{Q}^{x}: x \in[0, l)\right)$ on $W$ under which the coordinate process is an $L_{m}$-diffusion with $x=0$ a trap and with $x=l$ also a trap if $x=l$ is absorbing, i.e., the Markovian family with the transition probability given by $q(t, x, y) d m(y)$.

For $a \in[0, \infty)$, let $\pi_{a}$ be the first-passage time to $a$ :

$$
\pi_{a}(w)=\inf \{t \geq 0: w(t)=a\}, \quad w \in W .
$$


Lemma 4.1. The Laplace transform of the law of $\pi_{0}$ is given by

$$
\boldsymbol{Q}^{x}\left[e^{-\lambda \pi_{0}}\right]=\int_{0}^{\infty} e^{-\lambda t} \Pi(t, x) d t, \quad \lambda>0, x \in(0, l) .
$$

In particular, for $x \in(0, l)$, the law of $\pi_{0}$ under $\boldsymbol{Q}^{x}$ is given by

$$
\boldsymbol{Q}^{x}\left(\pi_{0} \in d t\right)=\Pi(t, x) d t \quad \text { on }(0, \infty)
$$

and the probability that the path hits the origin is given by

$$
\boldsymbol{Q}^{x}\left(\pi_{0}<\infty\right)=\int_{0}^{\infty} \Pi(t, x) d t=1-\frac{x}{l} .
$$

Proof. It is well-known that

$$
\boldsymbol{Q}^{x}\left[e^{-\lambda \pi_{y}}\right]=\frac{g_{\lambda}(x)}{g_{\lambda}(y)}, \quad \lambda>0,0<y<x<l .
$$

Letting $y \rightarrow 0+$, we obtain

$$
\boldsymbol{Q}^{x}\left[e^{-\lambda \pi_{0}}\right]=g_{\lambda}(x), \quad \lambda>0, x \in(0, l) .
$$

On the other hand,

$$
G(\lambda, x, y)=\int_{0}^{\infty} e^{-\lambda t} q(t, x, y) d t=g_{\lambda}(x) \psi_{\lambda}(y), \quad \lambda>0,0<y<x<l .
$$

Differentiating the second and the third terms with respect to $y$ and letting $y \rightarrow 0$, we obtain

$$
\int_{0}^{\infty} e^{-\lambda t} \Pi(t, x) d t=g_{\lambda}(x), \quad x \in(0, l) .
$$

Combining (4.9) and (4.11), we obtain (4.5). Letting $\lambda \rightarrow 0+$, we obtain

$$
\boldsymbol{Q}^{x}\left(\pi_{0}<\infty\right)=\int_{0}^{\infty} \Pi(t, x) d t=g_{0+}(x)=x \int_{x}^{l} \frac{d y}{y^{2}}=1-\frac{x}{l}, \quad x \in(0, l) .
$$

This completes the proof. 


\section{$\S 4.3$. The space of excursions}

Let $E$ be the totality of continuous paths $e \in W$ with lifetime $\zeta(e) \in(0, \infty)$ such that the following hold:

(i) $e(t)>0$ for any $0<t<\zeta(e)$.

(ii) $e(t)=0$ for $t \geq \zeta(e)$.

Denote

$$
\mathcal{E}=\{\Gamma \cap E: \Gamma \in \mathcal{W}\} \quad \text { and } \quad \mathcal{E}_{t}=\left\{\Gamma \cap E: \Gamma \in \mathcal{W}_{t}\right\}, t>0 .
$$

Then $\mathcal{E}$ (resp. $\mathcal{E}_{t}$ for $t>0$ ) coincides with the $\sigma$-field generated by the $\pi$-system which consists of cylinder sets given by

$$
C=\left\{e \in E: e\left(t_{1}\right) \in A_{1}, \ldots, e\left(t_{n}\right) \in A_{n}\right\}
$$

for some $0=t_{0}<t_{1}<\cdots<t_{n}<\infty$ (resp. $0<t_{1}<\cdots<t_{n}<t$ ) and $A_{1}, \ldots, A_{n} \in \mathcal{B}((0, \infty))$. Note that $C$ is included in the event $\left\{\zeta>t_{n}\right\}$, since $e\left(t_{n}\right)>0$ on $C$.

Suppose that

$$
l=\infty .
$$

Then Lemma 4.1 implies that the probability measure $\boldsymbol{Q}^{x}$ for $x \in(0, \infty)$ is concentrated on the space $E$.

Remark 7. The $\sigma$-field $\mathcal{E}$ is also generated by the $\pi$-system which consists of

$$
\left(\mathfrak{X}_{s}^{+}\right)^{-1}(C)
$$

for a cylinder set $C$ of the form (4.14) and $s>0$. Here the map $\mathfrak{X}_{s}^{+}$will be introduced in Section 5.1.

\section{$\S 5$. Stopping Times and the $\sigma$-Fields}

\section{$\S 5.1$. The $\sigma$-fields before and after a stopping time}

Let $\tau: E \rightarrow[0, \infty]$ be a random time. Set

$$
E^{\tau}=\{\tau<\zeta\}
$$


and

$$
\mathcal{E}^{\tau}=\left\{\Gamma \cap E^{\tau}: \Gamma \in \mathcal{E}\right\}, \quad \mathcal{E}_{t}^{\tau}=\left\{\Gamma \cap E^{\tau}: \Gamma \in \mathcal{E}_{t}\right\}, t \geq 0 .
$$

Define two measurable maps $\mathfrak{X}_{\tau}^{-}: E^{\tau} \rightarrow W$ and $\mathfrak{X}_{\tau}^{+}: E^{\tau} \rightarrow E$ by

$$
\mathfrak{X}_{\tau}^{-}(e)=e(\tau \wedge \cdot), \quad \mathfrak{X}_{\tau}^{+}(e)=e(\tau+\cdot) .
$$

Instead of $\mathfrak{X}_{\tau}^{-}$, we adopt a measurable map $\mathfrak{Y}_{\tau}^{-}: E^{\tau} \rightarrow E$ defined by

$$
\mathfrak{Y}_{\tau}^{-}(e)(t)= \begin{cases}e(t) & \text { if } 0 \leq t \leq \tau(e), \\ e(\tau(e))(1+\tau(e)-t) & \text { if } \tau(e)<t<\tau(e)+1, \\ 0 & \text { if } t \geq \tau(e)+1\end{cases}
$$

The reason why we prefer $\mathfrak{Y}_{\tau}^{-}$to $\mathfrak{X}_{\tau}^{-}$is that it is convenient for dealing with the last-exit time: See Lemma 5.3 (i).

Define

$$
\mathcal{E}_{(0, \tau)}=\left(\mathfrak{Y}_{\tau}^{-}\right)^{-1}(\mathcal{E}) \quad \text { and } \quad \mathcal{E}_{(\tau, \zeta)}=\left(\mathfrak{X}_{\tau}^{+}\right)^{-1}(\mathcal{E}) .
$$

Lemma 5.1. Suppose that $\tau$ is a $\left(\mathcal{E}_{t}\right)$-stopping time, i.e.,

$$
\{\tau \leq t\} \in \mathcal{E}_{t} \quad \text { for } t \geq 0 .
$$

Then the following statements hold:

(i) For any $t \geq 0,\{\tau \leq t\} \cap\{\tau<\zeta\} \in \mathcal{E}_{t}^{\tau}$.

(ii) $\tau(e)=\tau\left(\mathfrak{Y}_{\tau}^{-}(e)\right)$ for any $e \in E^{\tau}$.

(iii) For any $\Xi \in \mathcal{E}$,

$$
\left(\mathfrak{X}_{\tau}^{+}\right)^{-1}(\Xi)=\left\{e_{1}{ }^{\tau} e_{2}: e_{1} \in E^{\tau}, e_{2} \in \Xi, e_{2}(0)=e_{1}\left(\tau\left(e_{1}\right)\right)\right\},
$$

where, for two paths $e_{1} \in E^{\tau}$ and $e_{2} \in E$ with $e_{2}(0)=e_{1}\left(\tau\left(e_{1}\right)\right)$, the joint path $e_{1} \stackrel{\tau}{*} e_{2} \in E^{\tau}$ is defined by

$$
e_{1} * e_{2}(t)= \begin{cases}e_{1}(t) & \text { if } t \leq \tau\left(e_{1}\right), \\ e_{2}\left(t-\tau\left(e_{1}\right)\right) & \text { if } t>\tau\left(e_{1}\right) .\end{cases}
$$

(iv) Suppose that the set $A^{\tau}=\left\{e(\tau(e)) \in[0, \infty): e \in E^{\tau}\right\}$ is Borel measurable. Then, for any $\Gamma \in \mathcal{E}_{(\tau, \zeta)}$ expressed by $\Gamma=\left(\mathfrak{X}_{\tau}^{+}\right)^{-1}(\Xi)$ for some $\Xi \in \mathcal{E}$, it holds that

$$
\mathfrak{X}_{\tau}^{+}(\Gamma)=\Xi \cap\left\{e \in E: e(0) \in A^{\tau}\right\} \in \mathcal{E} .
$$

(v) It holds that

$$
\mathcal{E}=\sigma\left(\Gamma_{1} \cap \Gamma: \Gamma_{1} \in \mathcal{E}_{(0, \tau)}, \Gamma \in \mathcal{E}_{(\tau, \zeta)}\right)
$$


Proof. (i) This is clear by definition.

(ii) This is a direct consequence of Galmarino's theorem (see, e.g., [15, pp. 47, Exercise $\left.4.213^{\circ}\right]$ ).

(iii) Let $e \in\left(\mathfrak{X}_{\tau}^{+}\right)^{-1}(\Xi)$. Then $\mathfrak{X}_{\tau}^{+}(e) \in \Xi$. Since $e=e^{*}{ }^{*} \mathfrak{X}_{\tau}^{+}(e)$, e is contained in the RHS of (5.7). Conversely, let $e$ belong to the RHS of (5.7). Then $e=e_{1} * e_{2}$ for some $e_{1} \in E^{\tau}$ and $e_{2} \in \Xi$ with $e_{2}(0)=e_{1}\left(\tau\left(e_{1}\right)\right)$. Since $\tau(e)=\tau\left(e_{1}\right)$ by (ii), we obtain $\mathfrak{X}_{\tau}^{+}(e)=e_{2} \in \Xi$.

(iv) The equality is obvious by (iii). The measurability is obvious by the assumption.

(v) Let $\mathcal{E}^{\prime}$ denote the RHS of (5.10). Then the map

$$
E^{\tau} \ni e \mapsto\left(\mathfrak{Y}_{\tau}^{-}(e), \mathfrak{X}_{\tau}^{+}(e)\right) \in E \times E
$$

is $\mathcal{E}^{\prime} / \mathcal{E} \otimes \mathcal{E}$-measurable. For any $t>0$ and $A \in \mathcal{B}((0, l))$,

$$
\{e(t) \in A\} \cap\{\tau(e)>t\}=\left\{\mathfrak{Y}_{\tau}^{-}(e)(t) \in A\right\} \cap\left\{\tau\left(\mathfrak{Y}_{\tau}^{-}(e)\right)>t\right\} \in \mathcal{E}^{\prime}
$$

and

$\{e(t) \in A\} \cap\{\tau(e) \leq t\}=\left\{\mathfrak{X}_{\tau}^{+}(e)\left(t-\tau\left(\mathfrak{Y}_{\tau}^{-}(e)\right)\right) \in A\right\} \cap\left\{\tau\left(\mathfrak{Y}_{\tau}^{-}(e)\right) \leq t\right\} \in \mathcal{E}^{\prime}$.

Thus we obtain $\{e(t) \in A\} \in \mathcal{E}^{\prime}$ and therefore we obtain $\mathcal{E} \subset \mathcal{E}^{\prime}$.

\section{§5.2. The first-entrance and last-exit times and time reversal}

Let $a \in[0, l)$. Let $\tau_{a}$ be the first-entrance time to $[a, \infty)$ :

$$
\tau_{a}(w)=\inf \{t \geq 0: w(t) \geq a\}, \quad w \in W .
$$

Note that, if $e \in E$, then $\tau_{a}(e) \in[0, \zeta(e)]$ and $\tau_{0}(e)=0$. We define the last-exit time $\epsilon_{a}(e)$ from $[a, \infty)$ by

$$
\epsilon_{a}(e)= \begin{cases}\sup \{t \in[0, \zeta(e)]: e(t) \geq a\}, & e \in E^{\tau_{a}}=\left\{\tau_{a}(e)<\zeta(e)\right\} \\ \infty & e \in E \backslash E^{\tau_{a}}\end{cases}
$$

Note that $E^{\tau_{a}}=E^{\epsilon_{a}}$; in particular, if $a=0$, then $E^{\tau_{0}}=E^{\epsilon_{0}}=E$ and $\epsilon_{0}(e)=\zeta(e)$ for $e \in E$.

Let $x \in[0, l)$. We denote

$$
E^{x}=\{e \in E: e(0)=x\}
$$


and

$$
\mathcal{E}^{x}=\left\{\Gamma \cap E^{x}: \Gamma \in \mathcal{E}\right\}, \quad \mathcal{E}_{t}^{x}=\left\{\Gamma \cap E^{x}: \Gamma \in \mathcal{E}_{t}\right\}, t \geq 0 .
$$

For $e \in E^{0}$, we define the time reversal of the path $e$, which is denoted by $\check{e} \in E^{0}$, as

$$
\check{e}(t)=e\left((\zeta(e)-t)_{+}\right), \quad t \geq 0
$$

where $(x)_{+}=\max \{x, 0\}$. Note that

$$
\check{e}=e, \quad e \in E^{0} .
$$

For a set $\Gamma \in \mathcal{E}^{0}$, we define the time reversal of the set $\Gamma$, which is denoted by $\Gamma^{\vee} \in \mathcal{E}^{0}$, as

$$
\Gamma^{\vee}=\{\check{e}: e \in \Gamma\} .
$$

Note that

$$
\left(\Gamma^{\vee}\right)^{\vee}=\Gamma, \quad \Gamma \in \mathcal{E}^{0} .
$$

Lemma 5.2. Let $a \in(0, l)$ and $e \in E^{0}$. Then the following hold:

(i) $\tau_{a}(\check{e})=\zeta(e)-\epsilon_{a}(e)$.

(ii) $\epsilon_{a}(\check{e})=\zeta(e)-\tau_{a}(e)$.

(iii) $\zeta(\check{e})=\zeta(e)$.

(iv) $\epsilon_{a}(\check{e})-\tau_{a}(\check{e})=\epsilon_{a}(e)-\tau_{a}(e)$.

The proof is obvious, so that we omit it.

Let $a \in(0, l)$. We define a measurable map $\mathfrak{R}_{a}: E^{a} \rightarrow E^{a}$ by

$$
\mathfrak{R}_{a}(e)(t)= \begin{cases}e\left(\epsilon_{a}(e)-t\right) & \text { if } 0 \leq t<\epsilon_{a}(e), \\ e(t) & \text { if } t \geq \epsilon_{a}(e) .\end{cases}
$$

Note that

$$
\mathfrak{R}_{a} \circ \mathfrak{R}_{a}(e)=e, \quad e \in E^{a} .
$$

Lemma 5.3. Let $a \in(0, l)$. Then the following statements hold:

(i) $\epsilon_{a}\left(\mathfrak{Y}_{\epsilon_{a}}^{-}(e)\right)=\epsilon_{a}\left(\Re_{a}(e)\right)=\epsilon_{a}(e), e \in E^{a}$.

(ii) $\mathfrak{Y}_{\epsilon_{a}}^{-}\left(\mathfrak{R}_{a}(e)\right)=\mathfrak{R}_{a}\left(\mathfrak{Y}_{\epsilon_{a}}^{-}(e)\right), e \in E^{a}$. 
The proof is obvious, so that we omit it.

\section{§5.3. The $\sigma$-fields which represent the information of the path on the interval between two random times}

For $a \in[0, \infty)$, we denote

$$
E^{0, a}=\left\{e \in E: e(0)=0, \tau_{a}(e)<\zeta(e)\right\}
$$

and

$$
\mathcal{E}^{0, a}=\left\{\Gamma \cap E^{0, a}: \Gamma \in \mathcal{E}\right\}
$$

For $0 \leq x<a<\infty$, we note that

$$
0 \leq \tau_{x}(e)<\tau_{a}(e) \leq \epsilon_{a}(e)<\zeta(e), \quad e \in \mathcal{E}^{0, a} .
$$

We define

$$
\begin{gathered}
\mathcal{E}_{\left(\tau_{x}, \tau_{a}\right)}^{0, a}=\left(\mathfrak{Y}_{\tau_{a}}^{-} \circ \mathfrak{X}_{\tau_{x}}^{+}\right)^{-1}(\mathcal{E}) \cap \mathcal{E}^{0, a}, \\
\mathcal{E}_{\left(\tau_{a}, \zeta\right)}^{0, a}=\left(\mathfrak{X}_{\tau_{a}}^{+}\right)^{-1}(\mathcal{E}) \cap \mathcal{E}^{0, a}, \\
\mathcal{E}_{\left(\tau_{a}, \epsilon_{a}\right)}^{0, a}=\left(\mathfrak{Y}_{\epsilon_{a}}^{-} \circ \mathfrak{X}_{\tau_{a}}^{+}\right)^{-1}(\mathcal{E}) \cap \mathcal{E}^{0, a}
\end{gathered}
$$

and

$$
\mathcal{E}_{\left(\epsilon_{a}, \zeta\right)}^{0, a}=\left(\mathfrak{X}_{\epsilon_{a}}^{+} \circ \mathfrak{X}_{\tau_{a}}^{+}\right)^{-1}(\mathcal{E}) \cap \mathcal{E}^{0, a}
$$

Lemma 5.4. Let $a \in(0, l)$. Then the following statements hold:

(i) If $\Gamma \in \mathcal{E}_{\left(\tau_{a}, \zeta\right)}^{0, a}$ is expressed by $\Gamma=\left(\mathfrak{X}_{\tau_{a}}^{+}\right)^{-1}(\Xi)$ with $\Xi \in \mathcal{E}^{a}$, then $\mathfrak{X}_{\tau_{a}}^{+}(\Gamma)=$ $\Xi$.

(ii) If $\Gamma_{1}, \Gamma_{2} \in \mathcal{E}_{\left(\tau_{a}, \zeta\right)}^{0, a}$, then

$$
\mathfrak{X}_{\tau_{a}}^{+}\left(\Gamma_{1} \cap \Gamma_{2}\right)=\mathfrak{X}_{\tau_{a}}^{+}\left(\Gamma_{1}\right) \cap \mathfrak{X}_{\tau_{a}}^{+}\left(\Gamma_{2}\right) .
$$

(iii) If $\Gamma \in \mathcal{E}_{\left(\tau_{a}, \epsilon_{a}\right)}^{0, a}$, then $\Gamma^{\vee} \in \mathcal{E}_{\left(\tau_{a}, \epsilon_{a}\right)}^{0, a}$.

(iv) If $\Gamma \in \mathcal{E}_{\left(\tau_{a}, \epsilon_{a}\right)}^{0, a}$, then the following dichotomy holds:

$$
\left\{\tau_{a}=\epsilon_{a}\right\} \subset \Gamma \quad \text { or } \quad\left\{\tau_{a}=\epsilon_{a}\right\} \cap \Gamma=\emptyset .
$$


(v) $\Gamma \in \mathcal{E}_{\left(\epsilon_{a}, \zeta\right)}^{0, a}$ if and only if $\Gamma^{\vee} \in \mathcal{E}_{\left(0, \tau_{a}\right)}^{0, a}$.

(vi) $\mathcal{E}_{\left(\tau_{x}, \zeta\right)}^{0, x}$ is generated by $\mathcal{E}_{\left(\tau_{x}, \tau_{a}\right)}^{0, a}$ and $\mathcal{E}_{\left(\tau_{a}, \zeta\right)}^{0, a}$, i.e.,

$$
\mathcal{E}_{\left(\tau_{x}, \zeta\right)}^{0, x}=\sigma\left(\Gamma_{1} \cap \Gamma: \Gamma_{1} \in \mathcal{E}_{\left(\tau_{x}, \tau_{a}\right)}^{0, a}, \Gamma \in \mathcal{E}_{\left(\tau_{a}, \zeta\right)}^{0, a}\right)
$$

(vii) $\mathcal{E}_{\left(\tau_{a}, \zeta\right)}^{0, a}$ is generated by $\mathcal{E}_{\left(\tau_{a}, \epsilon_{a}\right)}^{0, a}$ and $\mathcal{E}_{\left(\epsilon_{a}, \zeta\right)}^{0, a}$, i.e.,

$$
\mathcal{E}_{\left(\tau_{a}, \zeta\right)}^{0, a}=\sigma\left(\Gamma_{2} \cap \Gamma_{3}: \Gamma_{2} \in \mathcal{E}_{\left(\tau_{a}, \epsilon_{a}\right)}^{0, a}, \Gamma_{3} \in \mathcal{E}_{\left(\epsilon_{a}, \zeta\right)}^{0, a}\right)
$$

Proof. (i) We can prove the claim similarly as we have done in Lemma 5.1 (iv). So we omit the proof.

(ii) For $i=1,2$, we can express the set $\Gamma_{i}$ by $\Gamma_{i}=\left(\mathfrak{X}_{\tau_{a}}^{+}\right)^{-1}\left(\Xi_{i}\right) \cap E^{0, a}$ with $\Xi_{i} \in \mathcal{E}^{a}$. By (i), we have $\mathfrak{X}_{\tau_{a}}^{+}\left(\Gamma_{i}\right)=\Xi_{i}$. Thus we obtain

$$
\begin{aligned}
\mathfrak{X}_{\tau_{a}}^{+}\left(\Gamma_{1} \cap \Gamma_{2}\right) & =\mathfrak{X}_{\tau_{a}}^{+}\left(\left(\mathfrak{X}_{\tau_{a}}^{+}\right)^{-1}\left(\Xi_{1}\right) \cap\left(\mathfrak{X}_{\tau_{a}}^{+}\right)^{-1}\left(\Xi_{2}\right)\right) \\
& =\mathfrak{X}_{\tau_{a}}^{+}\left(\left(\mathfrak{X}_{\tau_{a}}^{+}\right)^{-1}\left(\Xi_{1} \cap \Xi_{2}\right)\right) \\
& =\Xi_{1} \cap \Xi_{2} \\
& =\mathfrak{X}_{\tau_{a}}^{+}\left(\Gamma_{1}\right) \cap \mathfrak{X}_{\tau_{a}}^{+}\left(\Gamma_{2}\right) .
\end{aligned}
$$

(iii) $\Gamma \in \mathcal{E}_{\left(\tau_{a}, \epsilon_{a}\right)}^{0, a}$ is expressed by $\Gamma=\left(\mathfrak{X}_{\tau_{a}}^{+}\right)^{-1}\left(\left(\mathfrak{Y}_{\epsilon_{a}}^{-}\right)^{-1}(\Xi)\right) \cap E^{0, a}$ with $\Xi \in \mathcal{E}^{a}$. Then it is obvious that $\Gamma^{\vee}=\left(\mathfrak{X}_{\tau_{a}}^{+}\right)^{-1}\left(\left(\mathfrak{Y}_{\epsilon_{a}}^{-}\right)^{-1}\left(\mathfrak{R}_{a}(\Xi)\right)\right) \cap E^{0, a}$. Since $\mathfrak{R}_{a}(\Xi) \in \mathcal{E}^{a}$, we obtain $\Gamma^{\vee} \in \mathcal{E}_{\left(\tau_{a}, \epsilon_{a}\right)}^{0, a}$.

(iv) Let $\Gamma$ and $\Xi$ as above. Suppose that there exists an element $e \in \Gamma$ such that $e \in\left\{\tau_{a}=\epsilon_{a}\right\}$. Then the set $\Xi$ contains the element

$$
e_{a}=\mathfrak{Y}_{\epsilon_{a}}^{-} \circ \mathfrak{X}_{\tau_{a}}^{+}(e)=\left(a(1-t)_{+}: t \geq 0\right) .
$$

Thus $\Gamma$ contains the set

$$
\left(\mathfrak{X}_{\tau_{a}}^{+}\right)^{-1}\left(\left(\mathfrak{Y}_{\epsilon_{a}}^{-}\right)^{-1}\left(\left\{e_{a}\right\}\right)\right) \cap E^{0, a}=\left\{\tau_{a}=\epsilon_{a}\right\},
$$

which proves the claim.

(v) This is obvious.

(vi) Noting that

$$
\mathcal{E}_{\left(\tau_{x}, \tau_{a}\right)}^{0, a}=\left(\mathfrak{Y}_{\tau_{a}}^{-}\right)^{-1}\left(\mathcal{E}_{\left(\tau_{x}, \zeta\right)}^{0, x}\right) \cap \mathcal{E}^{0, a} \quad \text { and } \quad \mathcal{E}_{\left(\tau_{a}, \zeta\right)}^{0, a}=\left(\mathfrak{X}_{\tau_{a}}^{+}\right)^{-1}\left(\mathcal{E}_{\left(\tau_{x}, \zeta\right)}^{0, x}\right) \cap \mathcal{E}^{0, a},
$$

we can prove the claim similarly as we have done in Lemma $5.1(\mathrm{v})$. 
(vii) It suffices to show that the $\sigma$-field $\mathcal{E}^{a}$ is included in $\mathcal{E}^{\prime}$ which is generated by $\left(\mathfrak{Y}_{\epsilon_{a}}^{-}\right)^{-1}\left(\mathcal{E}^{a}\right)$ and $\left(\mathfrak{X}_{\epsilon_{a}}^{+}\right)^{-1}\left(\mathcal{E}^{a}\right)$.

Clearly, the map

$$
E^{a} \ni e \mapsto\left(\mathfrak{Y}_{\epsilon_{a}}^{-}(e), \mathfrak{X}_{\epsilon_{a}}^{+}(e)\right) \in E^{a} \times E^{a}
$$

is $\mathcal{E}^{\prime} / \mathcal{E}^{a} \otimes \mathcal{E}^{a}$-measurable. Let $\Xi=\left\{e \in \mathcal{E}^{a}: e(t) \in A\right\}$ for $t>0$ and $A \in \mathcal{B}((0, l))$. Then

$$
\Xi \cap\left\{t \leq \epsilon_{a}\right\}=\left\{e \in \mathcal{E}^{a}: \mathfrak{Y}_{\epsilon_{a}}^{-}(e)(t) \in A, \epsilon_{a}\left(\mathfrak{Y}_{\epsilon_{a}}^{-}(e)\right) \geq t\right\} \in \mathcal{E}^{\prime}
$$

and

$$
\Xi \cap\left\{t>\epsilon_{a}\right\}=\left\{e \in \mathcal{E}^{a}: \mathfrak{X}_{\epsilon_{a}}^{+}(e)(t) \in A, \epsilon_{a}\left(\mathfrak{Y}_{\epsilon_{a}}^{-}(e)\right)<t\right\} \in \mathcal{E}^{\prime} .
$$

Thus we obtain $\Xi \in \mathcal{E}^{\prime}$ and therefore we conclude that $\mathcal{E}^{a} \subset \mathcal{E}^{\prime}$, which proves the claim.

\section{§6. Proof of the Existence Theorem of the Excursion Measure Away from the Origin of $L_{m}$-Diffusion Processes}

Proof of Theorem 2.3. Following Ikeda-Watanabe's book [4], we start with the expression

$$
\boldsymbol{n}(\Gamma)=\int_{0}^{\infty} \boldsymbol{P}_{t}^{0,0}(\Gamma) \rho(t) d t, \quad \Gamma \in \mathcal{E}
$$

and show that $\boldsymbol{n}$ satisfies (2.27). Hence it suffices to show that

$$
\boldsymbol{n}\left(\left(\mathfrak{X}_{s}^{+}\right)^{-1}(C)\right)=\int_{(0, \infty)} d m\left(x_{0}\right) \Pi\left(s, x_{0}\right) \boldsymbol{Q}^{x_{0}}(C)
$$

for any cylinder set $C \in \mathcal{E}$ of the form (4.14) and for any $s>0$.

Let $t>t_{n}$ and $A \in \mathcal{B}((0, \infty))$. Then

$$
\begin{aligned}
(6.3)= & \int_{(0, \infty)} d \mu\left(x_{0}\right) p\left(s, 0, x_{0}\right) \int_{A_{1}} d \mu\left(x_{1}\right) p\left(t_{1}-t_{0}, x_{0}, x_{1}\right) \\
& \cdots \int_{A_{n}} d \mu\left(x_{n}\right) p\left(t_{n}-t_{n-1}, x_{n-1}, x_{n}\right) \int_{A} d \mu(x) p\left(t-t_{n}, x_{n}, x\right) \\
(6.4)= & \int_{(0, \infty)} d m\left(x_{0}\right) \Pi\left(s, x_{0}\right) \int_{A_{1}} d m\left(x_{1}\right) q\left(t_{1}-t_{0}, x_{0}, x_{1}\right) \\
& \cdots \int_{A_{n}} d m\left(x_{n}\right) q\left(t_{n}-t_{n-1}, x_{n-1}, x_{n}\right) \int_{A} d m(x) q\left(t-t_{n}, x_{n}, x\right) x \\
(6.5)= & \int_{(0, \infty)} d m\left(x_{0}\right) \Pi\left(s, x_{0}\right) \boldsymbol{Q}^{x_{0}}\left[\mathbf{1}_{C}(e) \mathbf{1}_{A}(e(t)) e(t)\right] .
\end{aligned}
$$


The expectation with respect to the probability $\boldsymbol{Q}^{x_{0}}$ is calculated by

$$
\begin{aligned}
& \boldsymbol{Q}^{x_{0}}\left[\mathbf{1}_{C}(e) \mathbf{1}_{A}(e(t)) e(t)\right] \\
= & \boldsymbol{Q}^{x_{0}}\left[\mathbf{1}_{C}(e) \boldsymbol{Q}^{x_{0}}\left[\mathbf{1}_{A}(e(t)) e(t) \mid \mathcal{W}_{t_{n}}\right]\right] \\
= & \int_{A} d m(x) x \boldsymbol{Q}^{x_{0}}\left[\mathbf{1}_{C}(e) q\left(t-t_{n}, e\left(t_{n}\right), x\right)\right] .
\end{aligned}
$$

Thus we obtain

$$
\begin{aligned}
& \boldsymbol{P}^{0}\left(\left(\mathfrak{X}_{s}^{+}\right)^{-1}(C \cap\{w(t) \in A\})\right) \\
= & \int_{A} d m(x) x \int_{(0, \infty)} d m\left(x_{0}\right) \Pi\left(s, x_{0}\right) \boldsymbol{Q}^{x_{0}}\left[\mathbf{1}_{C}(e) q\left(t-t_{n}, e\left(t_{n}\right), x\right)\right] .
\end{aligned}
$$

On the other hand,

$$
\begin{aligned}
\boldsymbol{P}^{0}\left(\left(\mathfrak{X}_{s}^{+}\right)^{-1}(\{w(t) \in A\})\right) & =\int_{A} d \mu(x) p(s+t, 0, x) \\
& =\int_{A} d m(x) \Pi(s+t, x) x .
\end{aligned}
$$

Combining it with (6.10), we obtain

$$
\begin{aligned}
\boldsymbol{P}_{s+t}^{0, x}\left(\left(\mathfrak{X}_{s}^{+}\right)^{-1}(C)\right) & =\boldsymbol{P}^{0}\left(\left(\mathfrak{X}_{s}^{+}\right)^{-1}(C) \mid w(s+t)=x\right) \\
& =\int_{(0, \infty)} d m\left(x_{0}\right) \Pi\left(s, x_{0}\right) \boldsymbol{Q}^{x_{0}}\left[\mathbf{1}_{C}(e) \frac{q\left(t-t_{n}, e\left(t_{n}\right), x\right)}{\Pi(s+t, x)}\right]
\end{aligned}
$$

for any $x \in(0, \infty)$. Letting $x \rightarrow 0+$, we obtain

$$
\boldsymbol{P}_{s+t}^{0,0}\left(\left(\mathfrak{X}_{s}^{+}\right)^{-1}(C)\right)=\int_{(0, \infty)} d m\left(x_{0}\right) \Pi\left(s, x_{0}\right) \boldsymbol{Q}^{x_{0}}\left[\mathbf{1}_{C}(e) \frac{\Pi\left(t-t_{n}, e\left(t_{n}\right)\right)}{\rho(s+t)}\right] .
$$

Now let us prove (6.1). Since $\left(\mathfrak{X}_{s}^{+}\right)^{-1}(C) \subset\left\{e\left(s+t_{n}\right)>0\right\}$, we have

$$
\begin{aligned}
\boldsymbol{n}\left(\left(\mathfrak{X}_{s}^{+}\right)^{-1}(C)\right) & =\int_{s+t_{n}}^{\infty} \boldsymbol{P}_{t}^{0,0}\left(\left(\mathfrak{X}_{s}^{+}\right)^{-1}(C)\right) \rho(t) d t \\
& =\int_{0}^{\infty} \boldsymbol{P}_{s+t_{n}+t}^{0,0}\left(\left(\mathfrak{X}_{s}^{+}\right)^{-1}(C)\right) \rho\left(s+t_{n}+t\right) d t \\
& =\int_{(0, \infty)} d m\left(x_{0}\right) \Pi\left(s, x_{0}\right) \boldsymbol{Q}^{x_{0}}\left[\mathbf{1}_{C}(e) \int_{0}^{\infty} \Pi\left(t, e\left(t_{n}\right)\right) d t\right] \\
& =\int_{(0, \infty)} d m\left(x_{0}\right) \Pi\left(s, x_{0}\right) \boldsymbol{Q}^{x_{0}}(C)
\end{aligned}
$$


Here we used the identity

$$
\int_{0}^{\infty} \Pi(t, x) d t=Q^{x}(\zeta \in(0, \infty))=1
$$

which is ensured by Lemma 4.1. The proof is completed.

Since (6.2) equals to (6.5), we obtain the following.

Corollary 6.1. Let $t>0$ and $s>0$. For any non-negative $\mathcal{E}_{t}$-measurable function $F$ and any non-negative Borel measurable function $G$ on $(0, \infty)$, it holds that

$$
\boldsymbol{P}^{0}\left[F \circ \mathfrak{X}_{s}^{+}(w) \cdot G(w(s+t))\right]=\int_{(0, \infty)} d m\left(x_{0}\right) \Pi\left(s, x_{0}\right) \boldsymbol{Q}^{x_{0}}[F(e) G(e(t)) e(t)] .
$$

\section{$\S 7$. Proof of the Decomposition Formulae}

\section{§7.1. Proof of the strong Markov property}

Proof of Theorem 2.5. It suffices to prove the claim in the case where $\tau$ is a constant time $t>0$. In fact, the proof in the general case is obtained by the continuity of the paths and the Feller property of the $L_{m}$-diffusion process.

Let $s>0$. Applying (6.1), the Markov property of the $L_{m}$-diffusion process and then Corollary 6.1, we obtain

$$
\begin{aligned}
(7.1) \boldsymbol{n}\left(\left(\mathfrak{X}_{s}^{+}\right)^{-1}\left(\Gamma_{1} \cap \Gamma\right)\right) & =\int_{(0, \infty)} d m\left(x_{0}\right) \Pi\left(s, x_{0}\right) \boldsymbol{Q}^{x_{0}}\left[\mathbf{1}_{\Gamma_{1}}(e) \mathbf{1}_{\Gamma}(e)\right] \\
& =\int_{(0, \infty)} d m\left(x_{0}\right) \Pi\left(s, x_{0}\right) \boldsymbol{Q}^{x_{0}}\left[\mathbf{1}_{\Gamma_{1}}(e) \boldsymbol{Q}^{e(t)}\left(\mathfrak{X}_{t}^{+}(\Gamma)\right)\right] \\
& =\boldsymbol{P}^{0}\left[\mathbf{1}_{\left(\mathfrak{X}_{s}^{+}\right)^{-1}\left(\Gamma_{1}\right)}(w) \cdot \frac{1}{w(s+t)} \cdot \boldsymbol{Q}^{w(s+t)}\left(\mathfrak{X}_{t}^{+}(\Gamma)\right)\right] .
\end{aligned}
$$

Letting $s \rightarrow 0+$, we obtain (2.39) in the case where $\tau$ is a constant time $t>0$.

Proof of Corollary 2.4. By Theorem 2.3, we obtain

$$
\boldsymbol{n}\left[e^{-\lambda \tau_{a}}\right]=\frac{1}{a} \boldsymbol{P}^{0}\left[e^{-\lambda \tau_{a}}\right]=\frac{\phi_{\lambda}(0)}{a \phi_{\lambda}(a)}=\frac{1}{\psi_{\lambda}(a)} .
$$

This proves (2.41). The equality (2.42) follows from the fact $\psi_{0}(x)=x$. 


\section{§7.2. Proof of the first-entrance-last-exit decomposition}

Proof of Theorem 2.6. By Theorem 2.5 and by Lemma 5.4 (ii), we obtain

$$
\boldsymbol{n}\left(\Gamma_{1} \cap \Gamma_{2} \cap \Gamma_{3}\right)=\frac{1}{a} \boldsymbol{P}^{0}\left(\Gamma_{1}\right) \boldsymbol{Q}^{a}\left(\mathfrak{X}_{\tau_{a}}^{+}\left(\Gamma_{2}\right) \cap \mathfrak{X}_{\tau_{a}}^{+}\left(\Gamma_{3}\right)\right) .
$$

In particular, if we take $\Gamma_{1}=E^{0, a}$, then we have

$$
\boldsymbol{n}\left(\Gamma_{2} \cap \Gamma_{3}\right)=\frac{1}{a} \boldsymbol{Q}^{a}\left(\mathfrak{X}_{\tau_{a}}^{+}\left(\Gamma_{2}\right) \cap \mathfrak{X}_{\tau_{a}}^{+}\left(\Gamma_{3}\right)\right) .
$$

By Corollary 2.2, we have

$$
\boldsymbol{n}\left(\left(\Gamma_{3}\right)^{\vee} \cap\left(\Gamma_{2}\right)^{\vee}\right)=\frac{1}{a} \boldsymbol{Q}^{a}\left(\mathfrak{X}_{\tau_{a}}^{+}\left(\Gamma_{2}\right) \cap \mathfrak{X}_{\tau_{a}}^{+}\left(\Gamma_{3}\right)\right) .
$$

By Lemma 5.4 (iii) and (v), we have $\left(\Gamma_{2}\right)^{\vee} \in \mathcal{E}_{\left(\tau_{a}, \epsilon_{a}\right)}^{0, a}$ and $\left(\Gamma_{3}\right)^{\vee} \in \mathcal{E}_{\left(0, \tau_{a}\right)}^{0, a}$. Thus, by Theorem 2.5 again, we have

$$
\boldsymbol{n}\left(\left(\Gamma_{3}\right)^{\vee} \cap\left(\Gamma_{2}\right)^{\vee}\right)=\frac{1}{a} \boldsymbol{P}^{0}\left(\left(\Gamma_{3}\right)^{\vee}\right) \boldsymbol{Q}^{a}\left(\mathfrak{X}_{\tau_{a}}^{+}\left(\left(\Gamma_{2}\right)^{\vee}\right)\right) .
$$

In particular, if we take $\Gamma_{3}=E^{0, a}$, then we have

$$
\boldsymbol{n}\left(\left(\Gamma_{2}\right)^{\vee}\right)=\frac{1}{a} \boldsymbol{Q}^{a}\left(\mathfrak{X}_{\tau_{a}}^{+}\left(\left(\Gamma_{2}\right)^{\vee}\right)\right) .
$$

By Corollary 2.2 again, we have

$$
\boldsymbol{Q}^{a}\left(\mathfrak{X}_{\tau_{a}}^{+}\left(\left(\Gamma_{2}\right)^{\vee}\right)\right)=\boldsymbol{Q}^{a}\left(\mathfrak{X}_{\tau_{a}}^{+}\left(\Gamma_{2}\right)\right) .
$$

Combining (7.5), (7.7), (7.8) and (7.10), we obtain the desired result.

\section{§7.3. Proof of the generalized Williams description}

Before proving Theorem 2.4, we prove Lemma 2.1.

Proof of Lemma 2.1. Let $0<b<a<\infty$. Since $\{M \geq a\} \in \mathcal{E}_{\left(\tau_{b}, \epsilon_{b}\right)}^{0, a}$ and $\mathfrak{X}_{\tau_{b}}^{+}(\{M \geq a\})=\left\{e \in E^{b}: M(e) \geq a\right\}$, Theorem 2.6 implies that

$$
\boldsymbol{n}(M \geq a)=\frac{1}{b} \boldsymbol{Q}^{b}(M \geq a) .
$$

Now we note that

$$
\boldsymbol{Q}^{b}\left[e^{-\lambda \tau_{a}}\right]=\frac{\psi_{\lambda}(b)}{\psi_{\lambda}(a)}, \quad \lambda>0,0<b<a<\infty .
$$


Letting $\lambda \rightarrow 0$, we have

$$
\boldsymbol{Q}^{b}(M \geq a)=\boldsymbol{Q}^{b}\left(\tau_{a}<\infty\right)=\frac{b}{a}, \quad 0<b<a<\infty .
$$

Combining (7.11) and (7.13), we obtain

$$
\boldsymbol{n}(M \geq a)=\frac{1}{a}
$$

This completes the proof.

Now we prove Theorem 2.4.

Proof of Theorem 2.4. It suffices to show that the Radon-Nikodym derivative is given by

$$
\frac{\boldsymbol{n}(\Gamma \cap\{M \in d a\})}{\boldsymbol{n}(M \in d a)}=\boldsymbol{R}^{a}(\Gamma)
$$

for any $\Gamma \in \mathcal{E}^{0}$.

Let $a \in(0, \infty)$ be fixed. Let $x \in(0, a)$ and $\Gamma \in \mathcal{E}_{\left(\tau_{x}, \zeta\right)}^{0, x}$. Then the strong Markov property says that

$$
\boldsymbol{n}(\Gamma \cap\{M \in[a, b)\})=\frac{1}{x} \boldsymbol{Q}^{x}\left(\mathfrak{X}_{\tau_{x}}^{+}(\Gamma) \cap\{M \in[a, b)\}\right) .
$$

Since $\boldsymbol{n}(M \in[a, b))=\frac{1}{x} \boldsymbol{Q}^{x}(M \in[a, b))$, we have

$$
\frac{\boldsymbol{n}(\Gamma \cap\{M \in[a, b)\})}{\boldsymbol{n}(M \in[a, b))}=\boldsymbol{Q}^{x}\left(\mathfrak{X}_{\tau_{x}}^{+}(\Gamma) \mid M \in[a, b)\right) .
$$

Letting $b \rightarrow a+$, we have

$$
\frac{\boldsymbol{n}(\Gamma \cap\{M \in d a\})}{\boldsymbol{n}(M \in d a)}=\boldsymbol{Q}^{x}\left(\mathfrak{X}_{\tau_{x}}^{+}(\Gamma) \mid M=a\right), \quad \Gamma \in \mathcal{E}_{\left(\tau_{x}, \zeta\right)}^{0, x} .
$$

Let $\Gamma=\Gamma_{1} \cap \Gamma_{2} \cap \Gamma_{3}$ with $\Gamma_{1} \in \mathcal{E}_{\left(\tau_{x}, \tau_{a}\right)}^{0, a}, \Gamma_{2} \in \mathcal{E}_{\left(\tau_{a}, \epsilon_{a}\right)}^{0, a}$ and $\Gamma_{3} \in \mathcal{E}_{\left(\epsilon_{a}, \zeta\right)}^{0, a}$. Here we denote $\mathcal{E}_{\left(\tau_{x}, \tau_{a}\right)}^{0, a}=\left(\mathfrak{X}_{\tau_{a}}^{-} \circ \mathfrak{X}_{\tau_{x}}^{+}\right)^{-1}(\mathcal{W})$. For any $b \in(a, l)$, Theorem 2.6 and (7.16) imply that

$$
\frac{1}{x} \boldsymbol{Q}^{x}\left(\mathfrak{X}_{\tau_{x}}^{+}(\Gamma)\right)=\frac{1}{a} \boldsymbol{P}^{0}\left(\Gamma_{1}\right) \boldsymbol{Q}^{a}\left(\mathfrak{X}_{\tau_{a}}^{+}\left(\Gamma_{2}\right) \cap\{M \in[a, b)\}\right) \boldsymbol{P}^{0}\left(\Gamma_{3}^{\vee}\right) .
$$

Dividing both sides by $\frac{1}{x} \boldsymbol{Q}^{x}(M \in[a, b))=\frac{1}{a} \boldsymbol{Q}^{a}(M \in[a, b))$ and letting $b \rightarrow a+$, we have

$$
\boldsymbol{Q}^{x}\left(\mathfrak{X}_{\tau_{x}}^{+}(\Gamma) \mid M=a\right)=\boldsymbol{P}^{0}\left(\Gamma_{1}\right) \boldsymbol{Q}^{a}\left(\mathfrak{X}_{\tau_{a}}^{+}\left(\Gamma_{2}\right) \mid M=a\right) \boldsymbol{P}^{0}\left(\Gamma_{3}^{\vee}\right)
$$


Noting that the conditional probability $\boldsymbol{Q}^{a}(\cdot \mid M=a)$ is concentrated on the set $\{M=a\}$ and that the dichotomy of Lemma 5.4 (iv) holds, we obtain

$$
\boldsymbol{Q}^{x}\left(\mathfrak{X}_{\tau_{x}}^{+}(\Gamma) \mid M=a\right)=\boldsymbol{R}^{a}(\Gamma) .
$$

By Lemma 5.4 (vi) and (vii), we conclude that the equality (7.21) holds for any $\Gamma \in \mathcal{E}_{\left(\tau_{x}, \zeta\right)}^{0, x}$, and therefore conclude that

$$
\int_{\left(a_{1}, a_{2}\right)} \boldsymbol{n}(\Gamma \cap\{M \in d a\})=\int_{\left(a_{1}, a_{2}\right)} \boldsymbol{R}^{a}(\Gamma) \boldsymbol{n}(M \in d a)
$$

for $0<a_{1}<a_{2}<\infty$ and $\Gamma \in \mathcal{E}_{\left(\tau_{x}, \zeta\right)}^{0, x}$. Since both sides of (7.22) have masses only on $\mathcal{E}^{0, a_{1}}$ and $x \in(0, a)$ is arbitrary, we conclude that (7.22) holds for any $\Gamma \in \mathcal{E}$, which completes the proof.

\section{$\S 8$. Proofs of the Convergence Theorem}

Proof of Lemma 2.2. (i) Noting that

$$
\rho(m ; u)=\int_{(0, \infty)} e^{-u \xi} \xi \sigma^{*}(m ; d \xi)
$$

we have

$$
\int_{0}^{1}|f(u)|^{2} \rho(m ; u) d u=\int_{(0, \infty)} g(\xi) \frac{\sigma^{*}(m ; d \xi)}{1+\xi^{2}}
$$

where

$$
g(\xi)=\left(1+\xi^{2}\right) \xi \int_{0}^{1}|f(u)|^{2} e^{-u \xi} d u .
$$

It is clear that $g(\xi)$ is continuous on $(0, \infty)$ and that $g(0+)=0$. Since

$$
g(\xi) \leq C^{2}\left(1+\xi^{2}\right) \xi \int_{0}^{1} u^{2} e^{-u \xi} d u=\frac{C^{2}\left(1+\xi^{2}\right)}{\xi^{2}},
$$

we conclude that $g(\xi)$ is bounded on $(0, \infty)$, and hence we obtain (i).

(ii) It suffices to show that

$$
\boldsymbol{n}(m ;\{\zeta \geq 1\})<\infty .
$$

By Corollary 2.1, we have

$$
\begin{aligned}
\boldsymbol{n}(m ;\{\zeta \geq 1\}) & =\int_{1}^{\infty} \rho(m ; u) d u \\
& =\int_{(0, \infty)} e^{-\xi} \sigma^{*}(m ; d \xi) .
\end{aligned}
$$


This proves (ii).

(iii) This is obvious since $\left\{\boldsymbol{N}(m ;(0, t], \Gamma \cap\{\zeta<1\}), \Gamma \in \mathcal{E}^{0}\right\}$ and $\{\boldsymbol{N}(m ;(0, t]$, $\left.\Gamma \cap\{\zeta \geq 1\}), \Gamma \in \mathcal{E}^{0}\right\}$ are independent.

Proof of Theorem 2.7. (i) It suffices to show the convergence of the variances, i.e.,

$$
\int_{0}^{1}|f(u)|^{2} \rho\left(m_{n} ; u\right) d u \rightarrow \int_{0}^{1}|f(u)|^{2} \rho(m ; u) d u \quad \text { as } n \rightarrow \infty .
$$

Using (8.2), we can rewrite (8.8) as

$$
\int_{(0, \infty)} g(\xi) \frac{\sigma^{*}\left(m_{n} ; d \xi\right)}{1+\xi^{2}} \rightarrow \int_{(0, \infty)} g(\xi) \frac{\sigma^{*}(m ; d \xi)}{1+\xi^{2}} \quad \text { as } n \rightarrow \infty .
$$

Since the function $g(\xi)$ defined in (8.3) extends to a bounded continuous function on $[0, \infty)$, the convergence (8.9) is justified by Lemma 3.2.

(ii) Set $f^{+}(x)=\max \{f(x), 0\}$ and $f^{-}(x)=\max \{-f(x), 0\}$. To prove the claim, it suffices to show the convergence of the joint distribution of $\left(U_{2}\left[f^{+}\right]\right.$ $\left.\left(m_{n} ; t\right), U_{2}\left[f^{-}\right]\left(m_{n} ; t\right)\right)$. This is equivalent to the convergence of the characteristic functions of $U_{2}\left[\lambda^{+} f^{+}+\lambda^{-} f^{-}\right]\left(m_{n} ; t\right)$ for any $\lambda^{+}, \lambda^{-}>0$. Hence we may assume that $f$ is non-negative.

To prove the convergence in law, we shall show the convergence of the corresponding Laplace transforms. Let $\lambda>0$ be fixed. By (2.47) and by Corollary 2.1, the Laplace transform of the law of $U_{2}[f](m ; t)$ is computed as

$$
\begin{aligned}
\boldsymbol{P}\left[\exp \left(-\lambda U_{2}[f](m ; t)\right)\right] & =\exp \left(-t \int_{\{\zeta \geq 1\}}\left(1-e^{-\lambda f(\zeta(e))}\right) \boldsymbol{n}(m ; d e)\right) \\
& =\exp \left(-t \int_{1}^{\infty}\left(1-e^{-\lambda f(u)}\right) \rho(m ; u) d u\right) \\
& =\exp \left(-t \int_{(0, \infty)} k(\xi) \frac{\sigma^{*}(m ; d \xi)}{1+\xi^{2}}\right),
\end{aligned}
$$

where

$$
k(\xi)=\left(1+\xi^{2}\right) \xi \int_{1}^{\infty}\left(1-e^{-\lambda f(u)}\right) e^{-u \xi} d u .
$$

By Lemma 3.2, it suffices to show that the function $k(\xi)$ extends to a bounded continuous function on $[0, \infty)$. 
It is obvious that $k(\xi)$ is continuous on $(0, \infty)$ by the dominated convergence. It is also obvious that $k(\xi)$ is bounded on $(0, \infty)$, since

$$
k(\xi) \leq\left(1+\xi^{2}\right) \xi \int_{1}^{\infty} e^{-u \xi} d u=\left(1+\xi^{2}\right) e^{-\xi}
$$

Since $\lim _{u \rightarrow \infty} f(u)=c$, we have

$$
k(0+)=\lim _{\xi \rightarrow 0+} \xi \int_{1}^{\infty}\left(1-e^{-\lambda f(u)}\right) e^{-u \xi} d u=1-e^{-\lambda c} .
$$

Here, if $c=\infty$, then the third expression is understood as 1 . Therefore we complete the proof.

Proof of Corollary 2.6. Since $m_{\lambda} \rightarrow m^{(\alpha)}$ in $\mathcal{M}_{1}$, we can apply Theorem 2.7. Therefore the only thing we have to prove is that

$$
U_{i}[f]\left(m_{\lambda} ; t\right) \stackrel{\text { law }}{=} U_{i}\left[f_{\lambda}\right](m ; \lambda t), \quad i=1,2 .
$$

This is equivalent to

$$
\rho\left(m_{\lambda} ; u\right)=\lambda^{\frac{1}{\alpha}+1} L(\lambda) \rho\left(m ; \lambda^{\frac{1}{\alpha}} L(\lambda) u\right) .
$$

This relation immediately follows from the fact that

$$
\rho(a m(b \cdot) ; u)=\frac{b^{2}}{a} \rho\left(m ; \frac{b}{a} u\right), \quad a, b>0 .
$$

\section{Acknowledgments}

I would like to express my sincerest gratitude to Professor Yoichiro Takahashi, who is my supervisor, for valuable guidance and hearty encouragement. I wish to extend my sincerest appreciation to Professor Shinzo Watanabe for stimulating discussions and warm encouragement. I am greatly thankful to Professors Yuji Kasahara and Shin'ichi Kotani, who allowed me to access the first drafts of their recent works and gave me a lot of valuable comments.

\section{References}

[1] Barlow, M., Pitman, J. and Yor, M., Une extension multidimensionnelle de la loi de l'arc sinus, Séminaire de Probabilités, XXIII, 294-314, Lecture Notes in Math., 1372, Springer, Berlin, 1989. 
[2] Blumenthal, R. M., Excursions of Markov processes, Probability and its Applications, Birkhäuser Boston Inc., Boston, MA, 1992.

[3] Dym, H. and McKean, H. P., Gaussian processes, function theory, and the inverse spectral problem, Probab. Math. Statist., 31, Academic Press, New York, 1976.

[4] Ikeda, N. and Watanabe, S., Stochastic differential equations and diffusion processes, North-Holland Mathematical Library, 24, North-Holland Publishing Co., Amsterdam, second edition, 1989.

[5] Itô, K., Poisson point processes attached to Markov processes, Proceedings of the Sixth Berkeley Symposium on Mathematical Statistics and Probability (Univ. California, Berkeley, Calif., 1970/1971), Vol. III: Probability theory, 225-239, Berkeley, Calif., 1972.

[6] Itô, K. and McKean, H. P., Jr, Diffusion processes and their sample paths, Second printing, corrected, Die Grundlehren der mathematischen Wissenschaften, 125, SpringerVerlag, Berlin, 1974.

[7] Kasahara, Y., Spectral theory of generalized second order differential operators and its applications to Markov processes, Japan. J. Math. (N.S.), 1 (1975/76), 67-84.

[8] Kasahara, Y. and Watanabe, S., Brownian representation of a class of Lévy processes and its application to occupation times of diffusion processes, to appear in Illinois J. Math., 2006.

[9] Kasahara, Y. and Yano, Y., On a generalized arc-sine law for one-dimensional diffusion processes, Osaka J. Math., 42 (2005), 1-10.

[10] Knight, F. B., Characterization of the Levy measures of inverse local times of gap diffusion, Seminar on Stochastic Processes, 1981 (Evanston, Ill., 1981), 53-78, Progr. Prob. Statist., 1, Birkhäuser Boston, Mass., 1981.

[11] Kotani, S., On a generalized Sturm-Liouville operator with a singular boundary, $J$. Math. Kyoto Univ., 15 (1975), 423-454.

[12] Short Krein spaces, Preprint.

[13] Kotani, S. and Watanabe, S., Kreı̆n's spectral theory of strings and generalized diffusion processes, Functional analysis in Markov processes (Katata/Kyoto, 1981), 235-259, Lecture Notes in Math., 923, Springer, Berlin, 1982.

[14] Minami, N., Ogura, Y. and Tomisaki, M., Asymptotic behavior of elementary solutions of one-dimensional generalized diffusion equations, Ann. Probab., 13 (1985), 698-715.

[15] Revuz, D. and Yor, M., Continuous martingales and Brownian motion, Grundlehren der Mathematischen Wissenschaften, 293, Springer-Verlag, Berlin, third edition, 1999.

[16] Rogers, L. C. G. and Williams, D., Diffusions, Markov processes, and martingales, Vol. 1, Foundations, Cambridge Mathematical Library, Cambridge University Press, Cambridge, 2000.

[17] — Diffusions, Markov processes, and martingales, Vol. 2, Itô calculus, Cambridge Mathematical Library, Cambridge University Press, Cambridge, 2000

[18] Watanabe, S., Generalized arc-sine laws for one-dimensional diffusion processes and random walks, Stochastic analysis (Ithaca, NY, 1993), 157-172, Proc. Sympos. Pure Math., 57, Amer. Math. Soc., Providence, RI, 1995.

[19] Watanabe, S., Yano, K. and Yano, Y., A density formula for the law of time spent on the positive side of one-dimensional diffusion processes, J. Math. Kyoto Univ., 45 (2005), 781-806.

[20] Williams, D., Path decomposition and continuity of local time for one-dimensional diffusions. I, Proc. London Math. Soc. (3), 28 (1974), 738-768.

[21] Yano, Y., On the occupation time on the half line of pinned diffusion processes, to appear in Publ. RIMS, Kyoto Univ. 